\title{
Synthesis and Catalytic Activity of Pincer-Type Bis(benzimidazolin-2-ylidene) Palladium Complexes
}

\author{
F. Ekkehardt Hahn, ${ }^{\star \dagger}$ Mareike C. Jahnke, ${ }^{\dagger}$ Valente Gomez-Benitez, David \\ Morales-Morales, ${ }^{*} \neq$ and Tania Pape ${ }^{\dagger}$ \\ Institut für Anorganische und Analytische Chemie der Westfälischen Wilhelms-Universität \\ Münster, Corrensstraße 36, D-48149 Münster, Germany, and \\ Instituto de Quimica, Universidad Nacional Autonoma de Mexico, Cd. Universitaria, \\ Circuito Exterior, Coyoacan, 04510 Mexico D.F., Mexico
}

\section{Supporting Information}

\author{
Page \\ Plots of the cations in $\mathbf{1}, \mathbf{2} \cdot 0.5 \mathrm{MeOH}, \mathbf{4} \cdot \mathrm{CH}_{2} \mathrm{Cl}_{2}$ and $\mathbf{6} \cdot \mathrm{MeOH}$




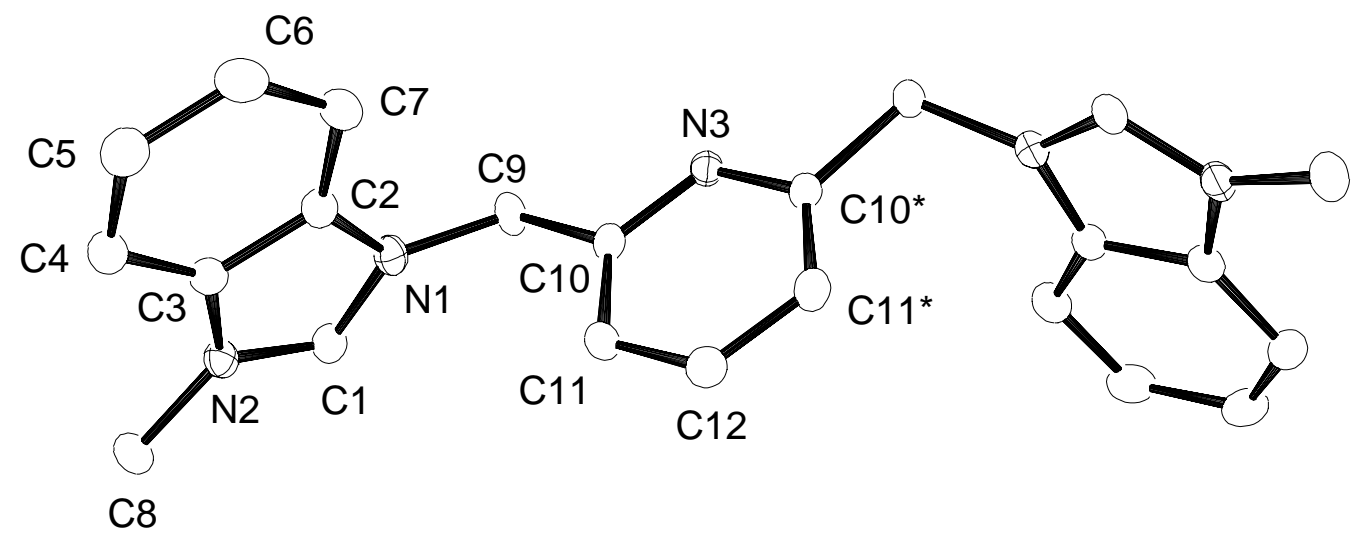

Molecular structure of the dication of $\mathbf{1}$. The molecule resides on a twofold axis which passes through C12-N3.

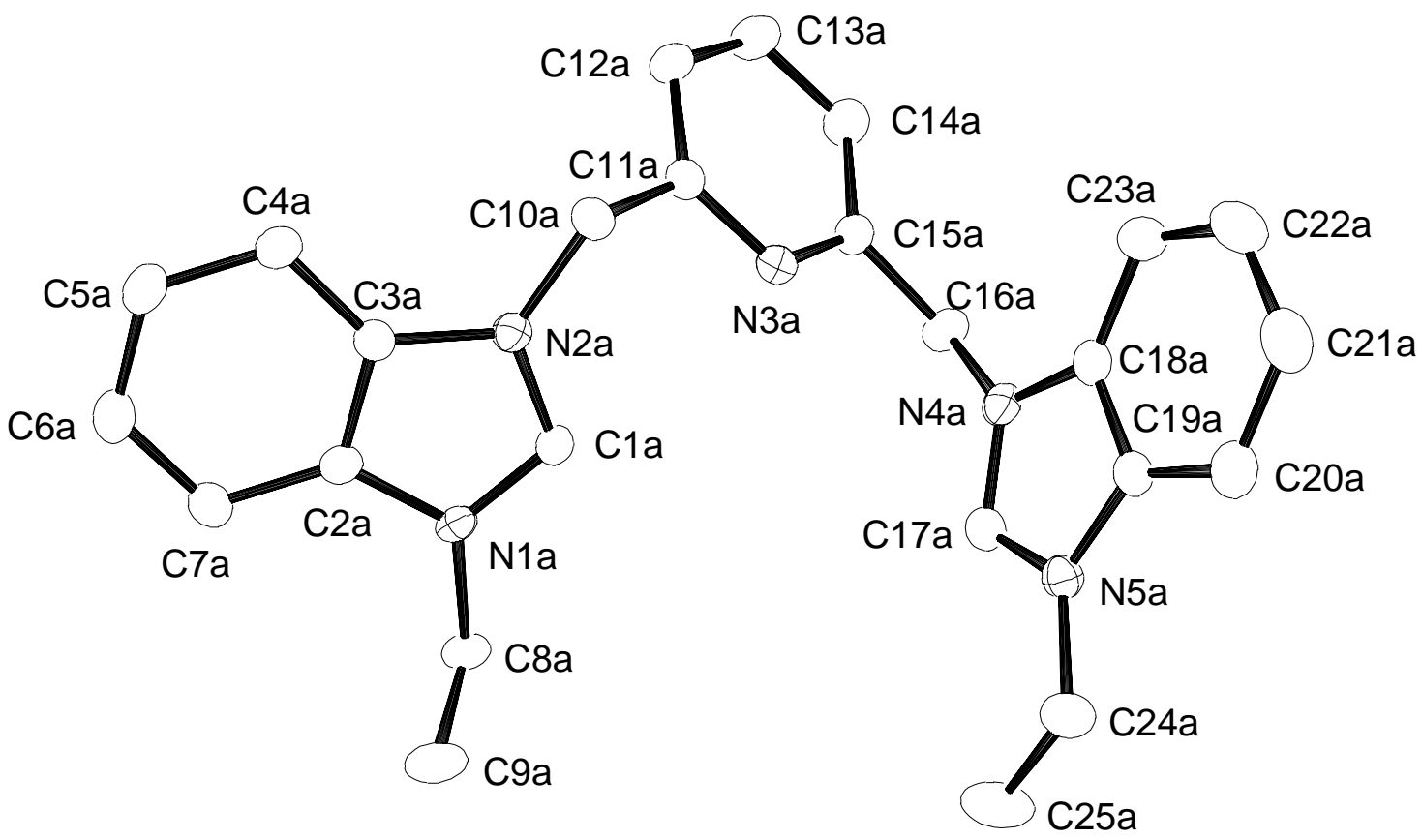

Molecular structure of the dication in $2 \cdot 0.5 \mathrm{MeOH}$. The asyymetric unit contains two identical molecules of $\mathbf{2}$ and one molecule of $\mathrm{MeOH}$. One of the four bromide anions in the asymmetric unit is disordered over two positions $(60 \% / 40 \%)$. 


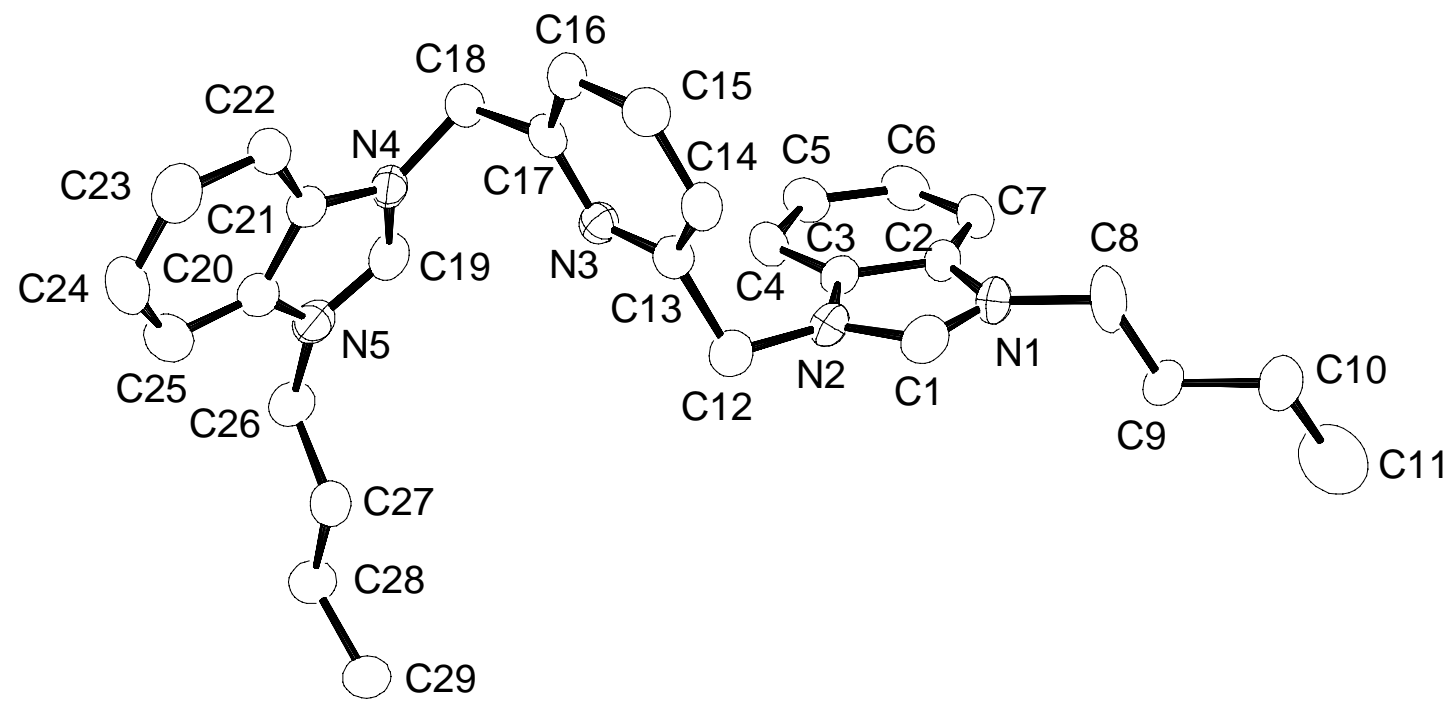

Molecular structure of the dication in $\mathbf{4} \cdot \mathrm{CH}_{2} \mathrm{Cl}_{2}$.

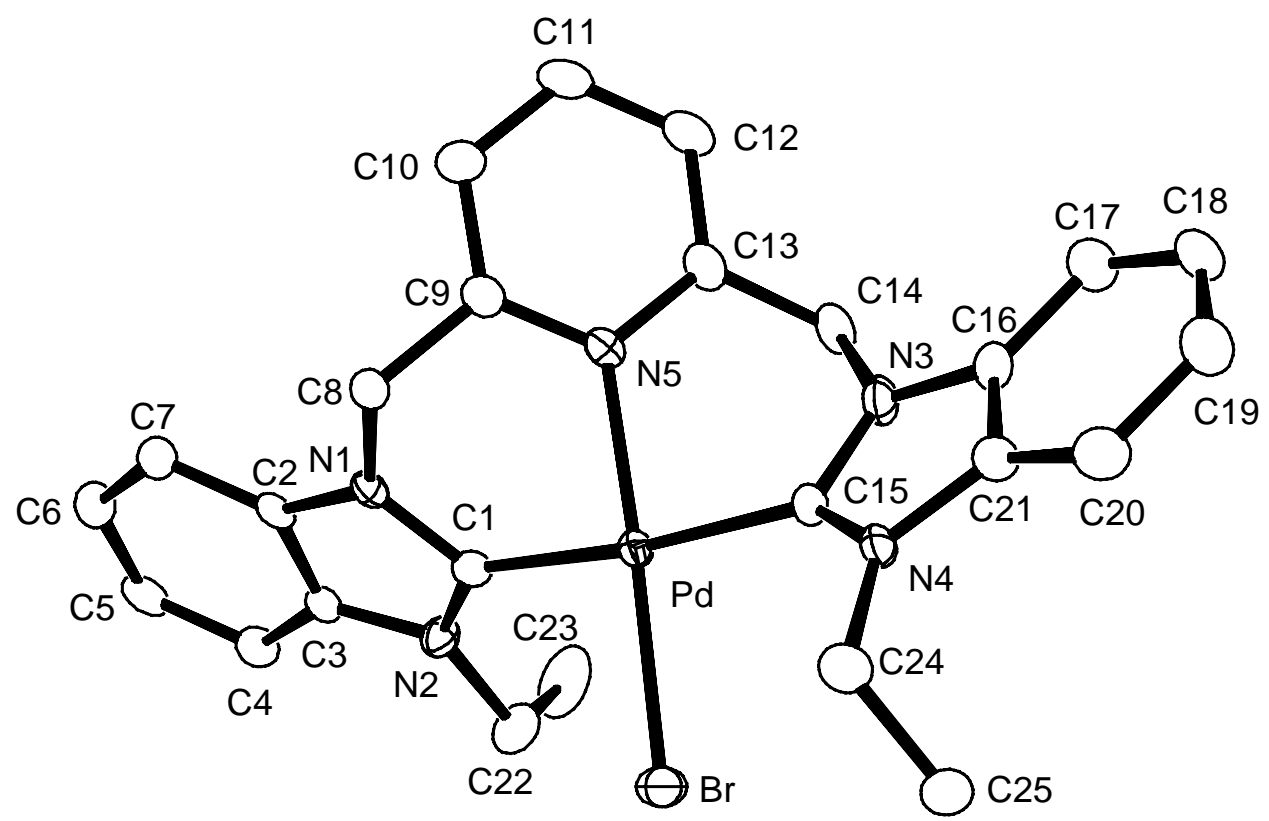

Molecular structure of the cation in $6 \cdot \mathrm{MeOH}$. 


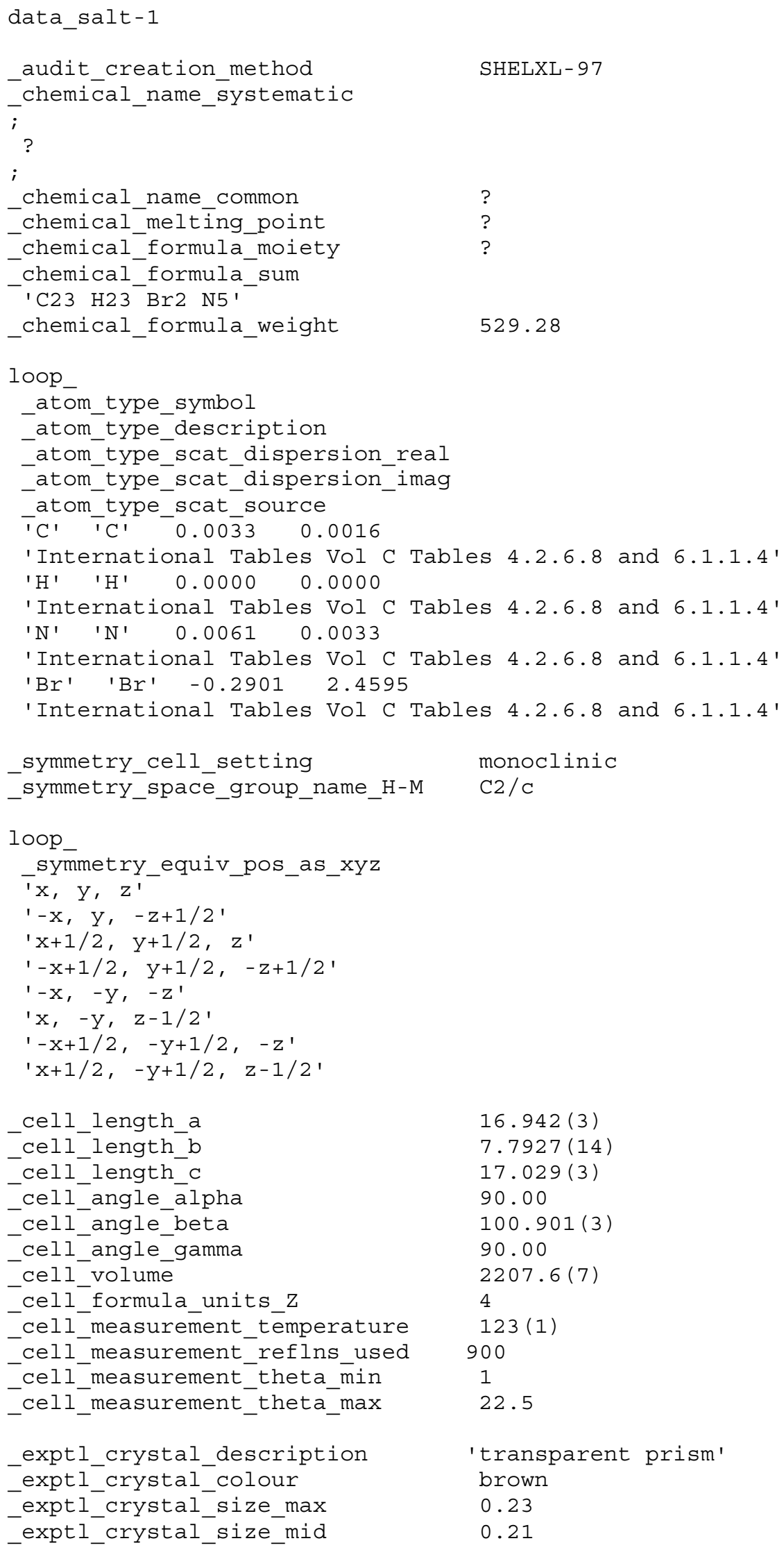




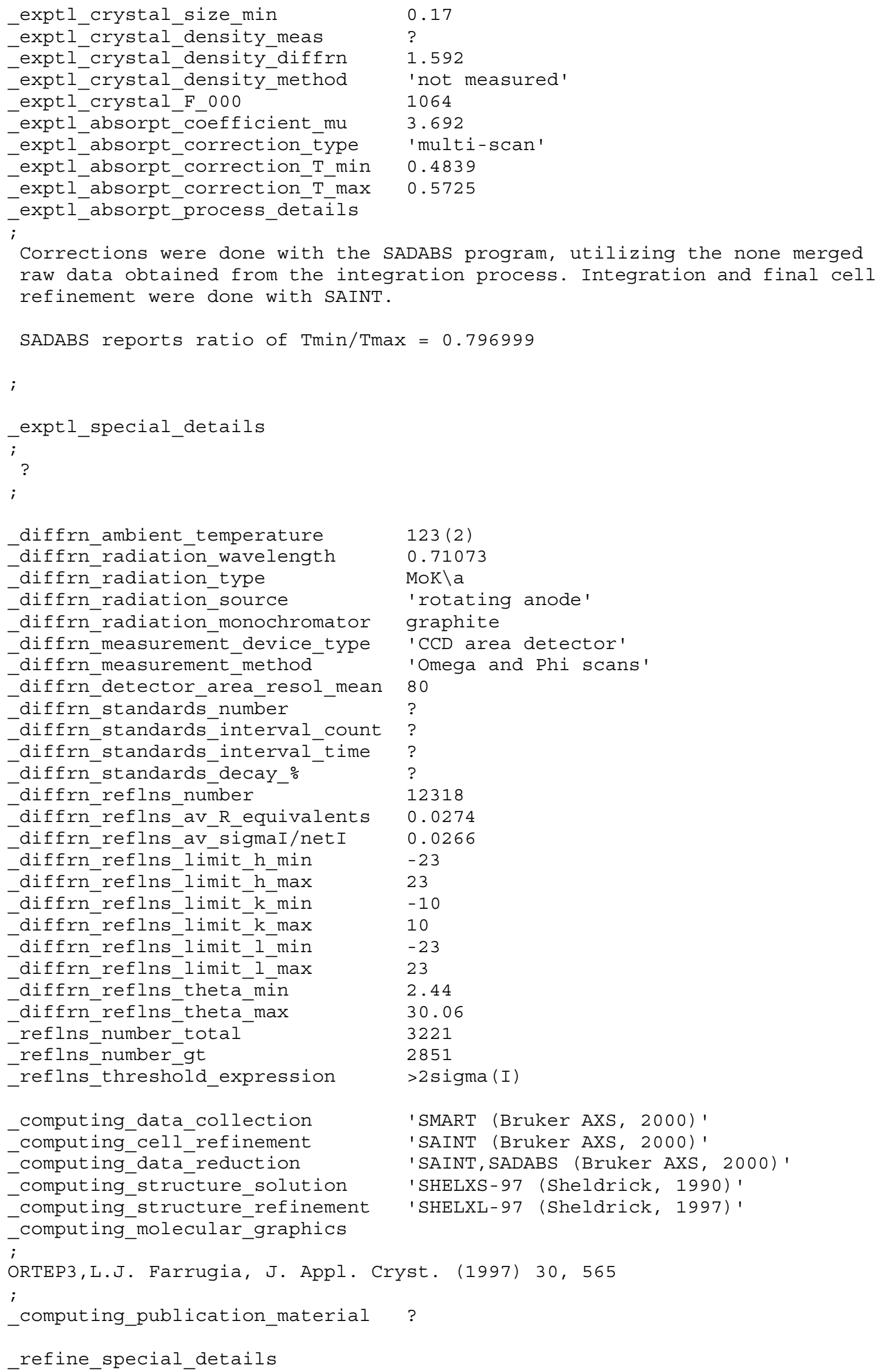


Refinement of $\mathrm{F}^{\wedge} 2^{\wedge}$ against $\mathrm{ALL}$ reflections. The weighted R-factor wR and goodness of fit $S$ are based on $\mathrm{F}^{\wedge} 2^{\wedge}$, conventional R-factors $\mathrm{R}$ are based on $F$, with $F$ set to zero for negative $F^{\wedge} 2^{\wedge}$. The threshold expression of $\mathrm{F}^{\wedge} 2^{\wedge}>2$ sigma $\left(\mathrm{F}^{\wedge} 2^{\wedge}\right)$ is used only for calculating R-factors (gt) etc. and is not relevant to the choice of reflections for refinement. R-factors based on $\mathrm{F}^{\wedge} 2^{\wedge}$ are statistically about twice as large as those based on $\mathrm{F}$, and $\mathrm{R}-$ factors based on ALL data will be even larger.

;

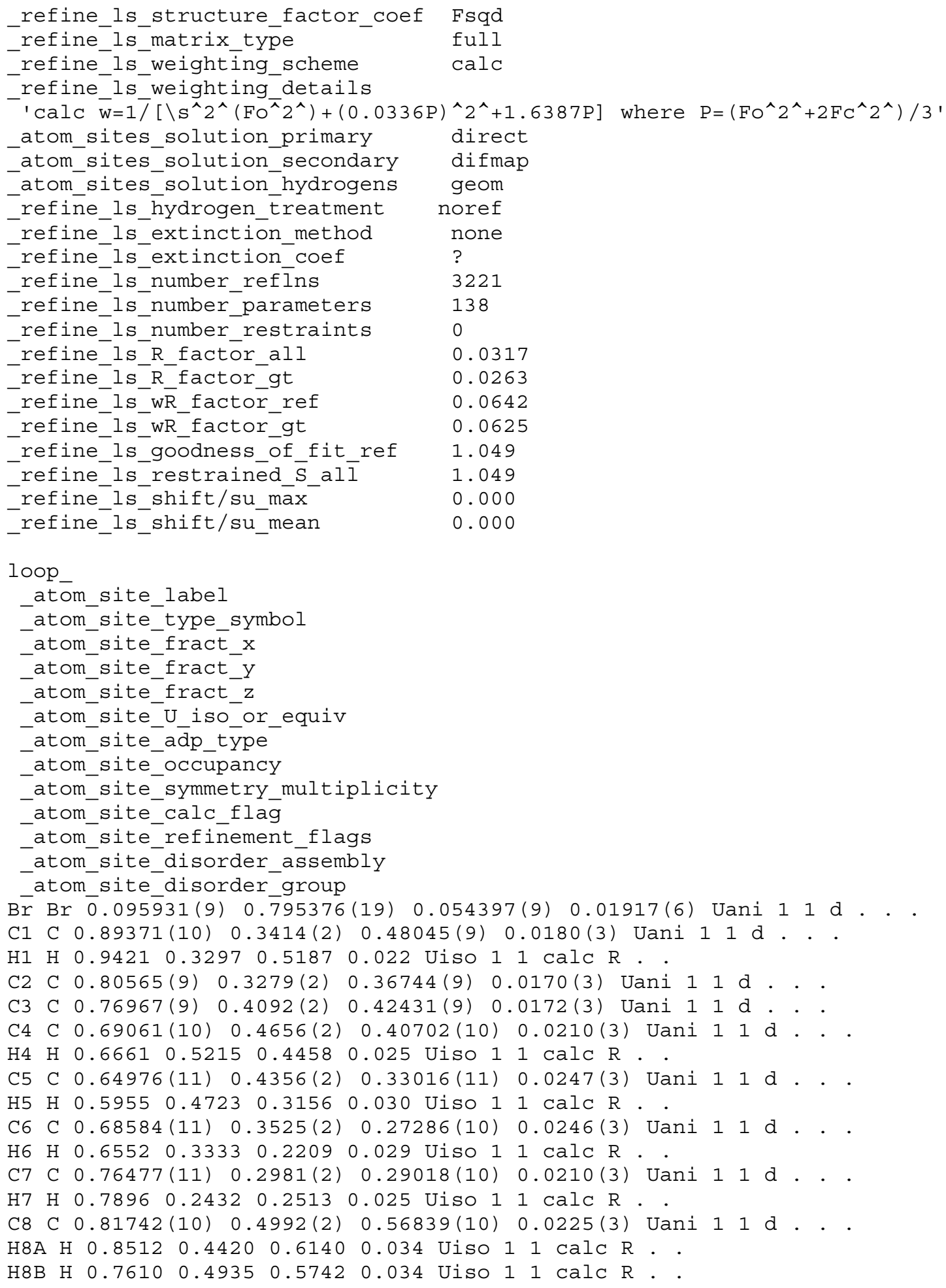


H8C H 0.83380 .61950 .56660 .034 Uiso 11 calc $\mathrm{R}$. .

C9 C $0.94321(10) 0.2017(2) \quad 0.36774(10) 0.0186(3)$ Uani 11 d. .

H9A H 0.92020 .09380 .34250 .022 Uiso 11 calc $\mathrm{R}$. .

H9B H 0.99020 .17140 .40950 .022 Uiso 11 calc $\mathrm{R}$.

C10 C $0.97083(9) \quad 0.3124(2) \quad 0.30524(9) \quad 0.0154(3)$ Uani 11 d . .

C11 C $0.96884(10) 0.4897(2) \quad 0.30733(10) 0.0192(3)$ Uani $11 d$.

H11 H $0.94670 .5483 \quad 0.3470 \quad 0.023$ Uiso 11 calc R . .

C12 C $1.00000 .5793(3) \quad 0.25000 .0211(4)$ Uani 12 d S . .

$\mathrm{H} 12 \mathrm{H} 1.0000 \quad 0.70130 .2500 \quad 0.025$ Uiso 12 calc SR. .

N1 N $0.88321(8) \quad 0.28769(17) \quad 0.40495(8) \quad 0.0174(3)$ Uani 11 d . .

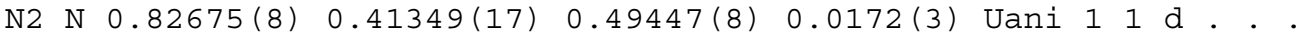

N3 N $1.0000 \quad 0.2232(2) \quad 0.2500 \quad 0.0150(3)$ Uani 12 d S . .

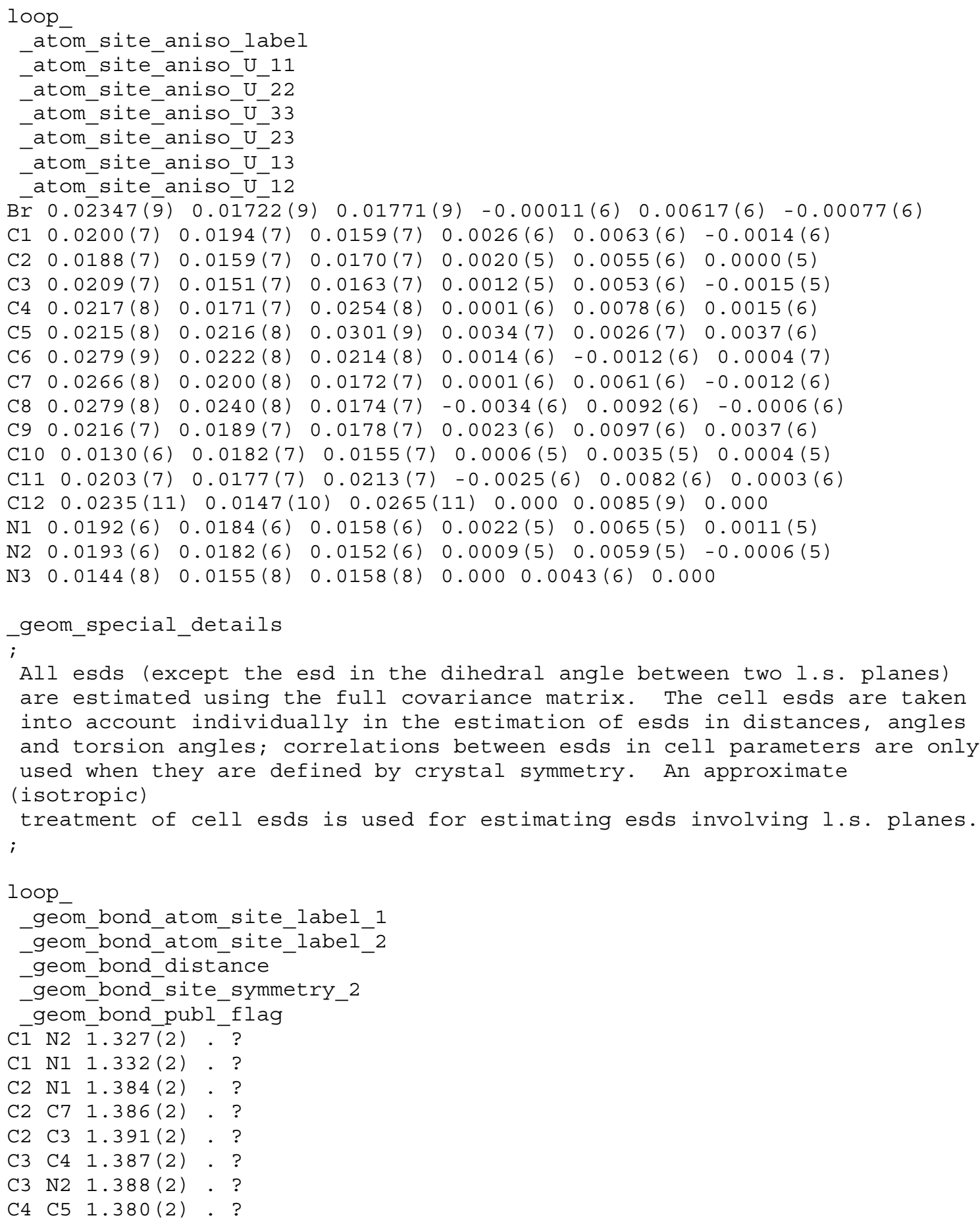




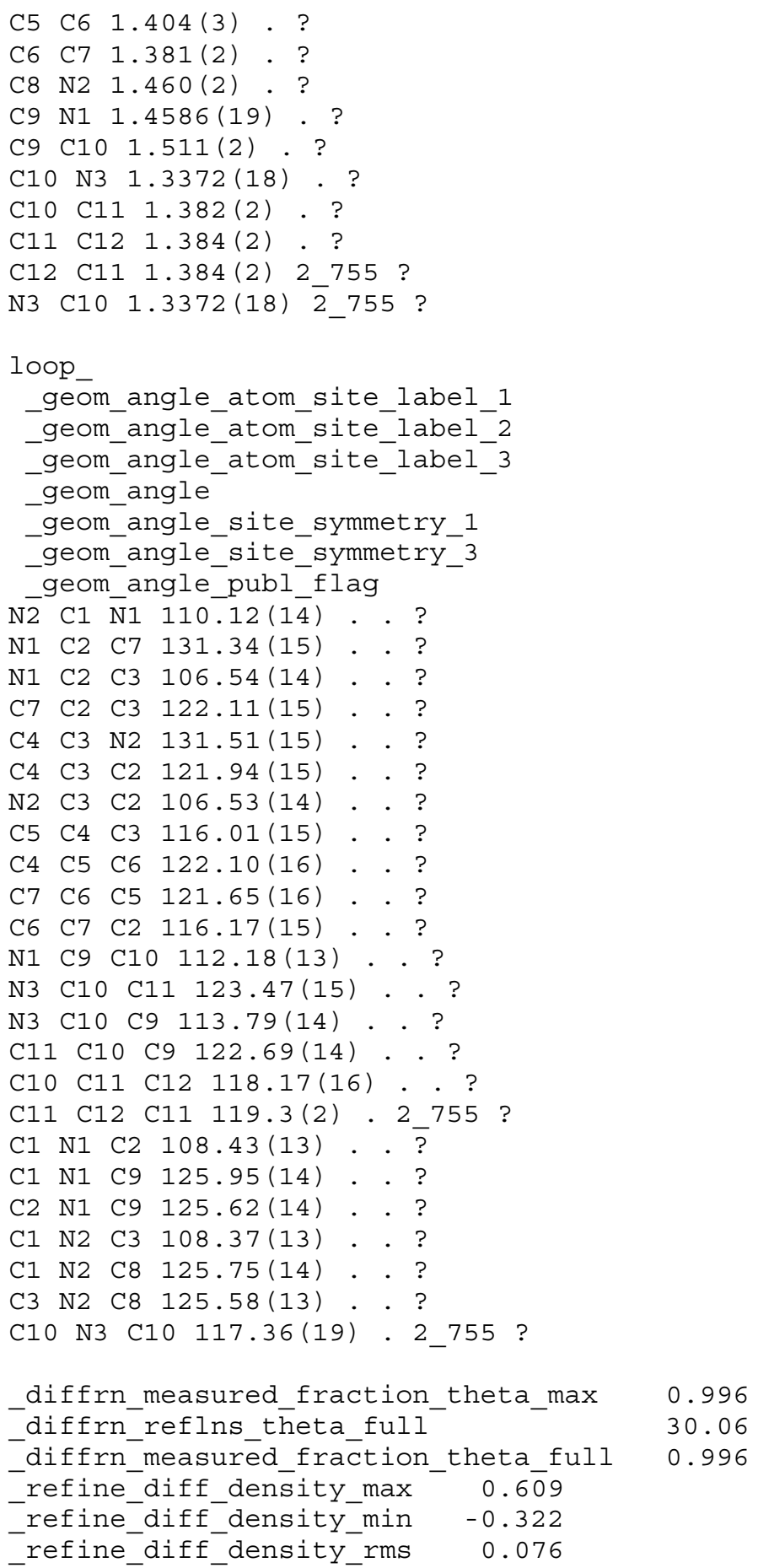




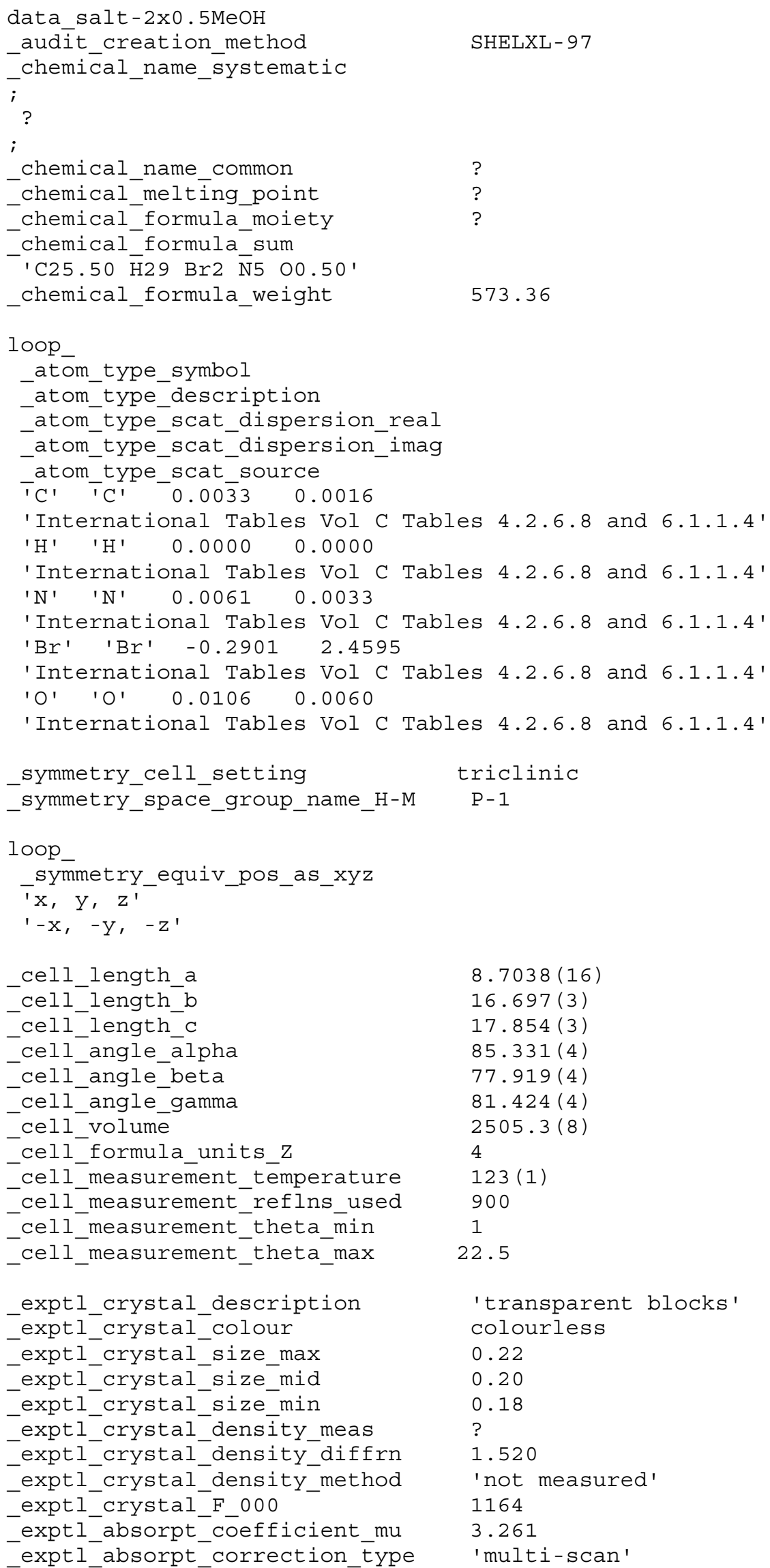




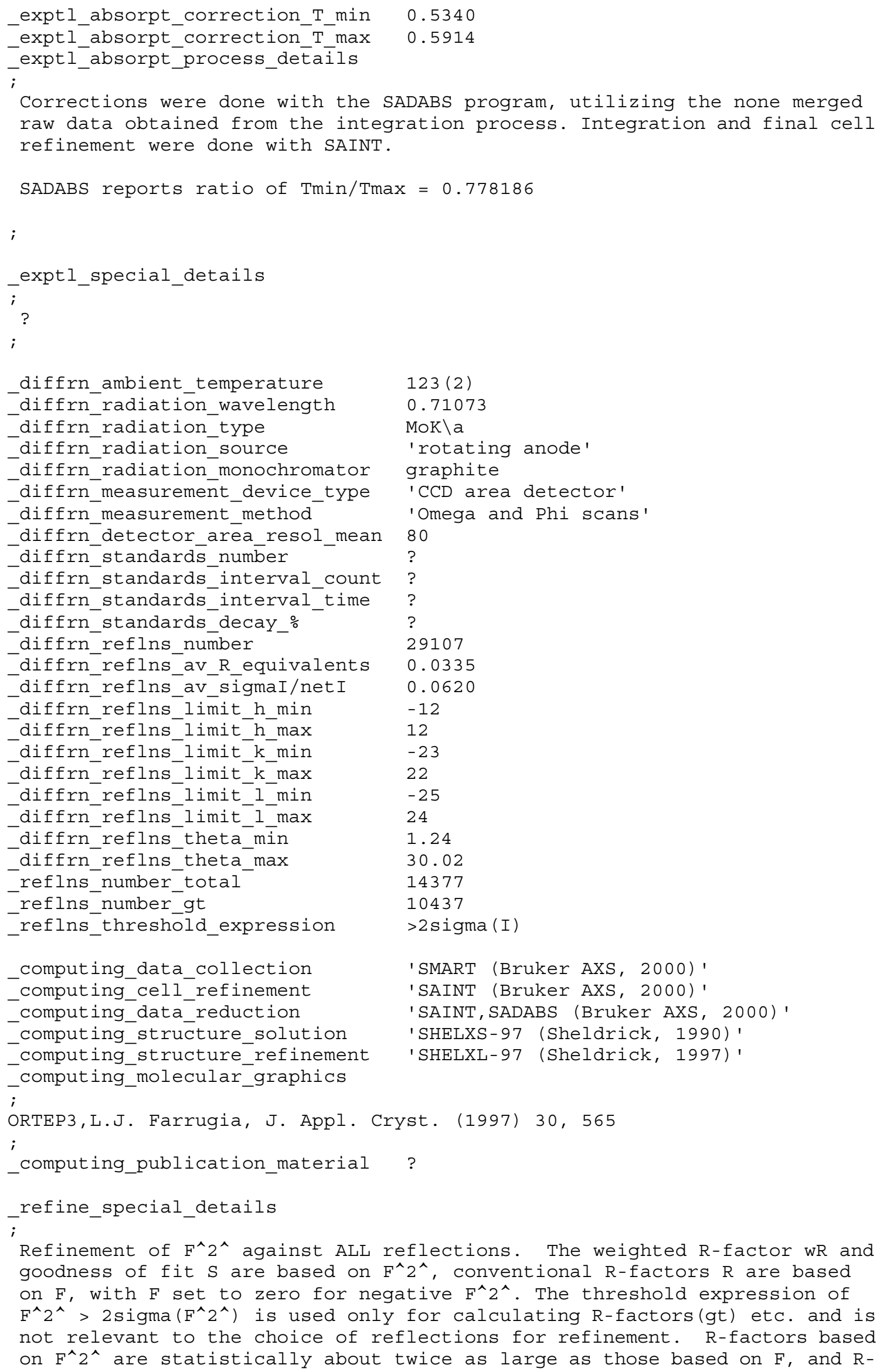
goodness of fit $S$ are based on $\mathrm{F}^{\wedge} 2^{\wedge}$, conventional R-factors $\mathrm{R}$ are based on $F$, with $F$ set to zero for negative $F^{\wedge} 2^{\wedge}$. The threshold expression of $\mathrm{F}^{\wedge} 2^{\wedge}>2 \mathrm{sigma}\left(\mathrm{F}^{\wedge} 2^{\wedge}\right)$ is used only for calculating R-factors (gt) etc. and is not relevant to the choice of reflections for refinement. R-factors based on $\mathrm{F}^{\wedge} 2^{\wedge}$ are statistically about twice as large as those based on $\mathrm{F}$, and $\mathrm{R}-$ 
factors based on ALL data will be even larger.

;

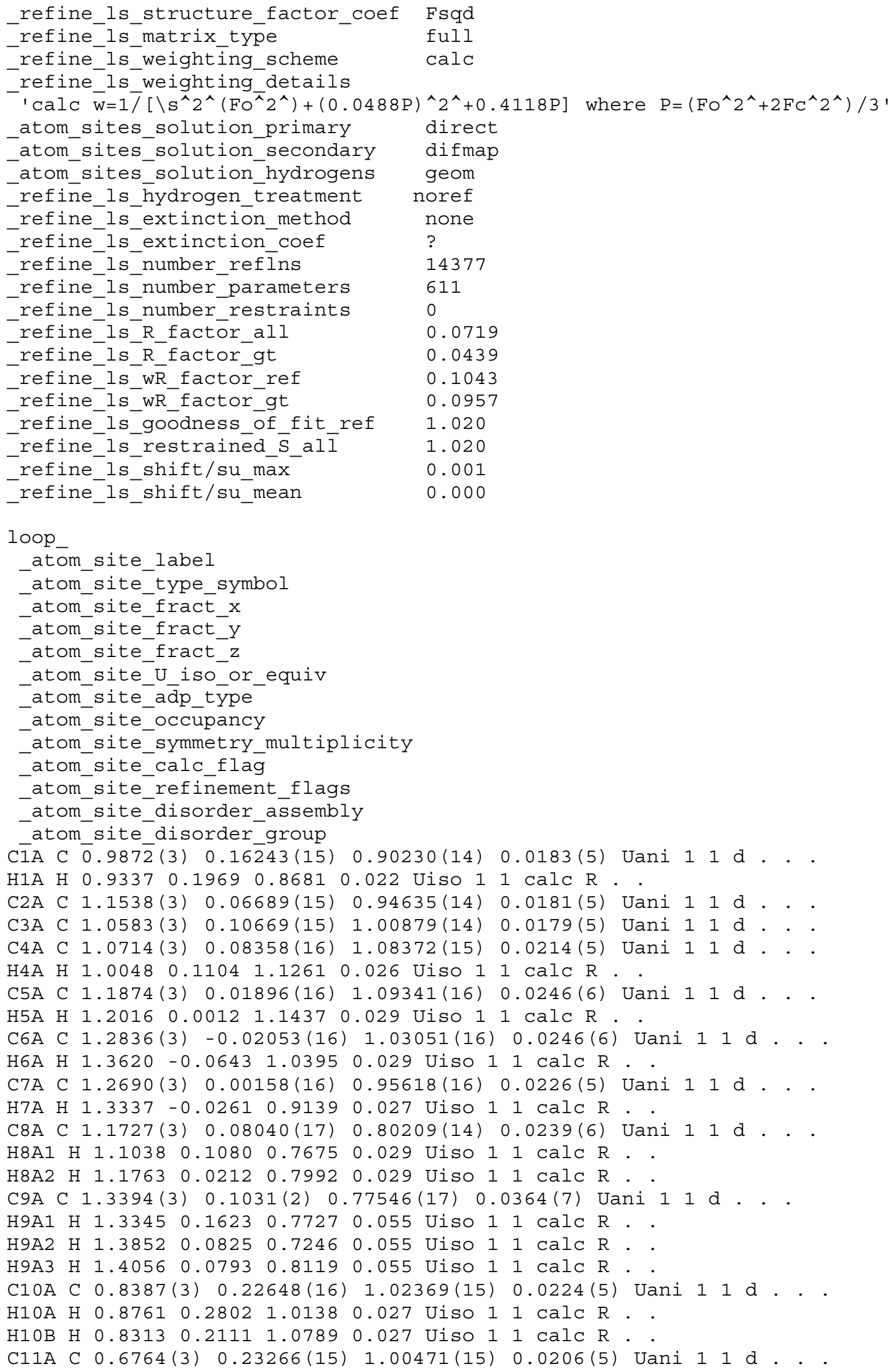


C12A C $0.5489(3) \quad 0.20988(17) \quad 1.05848(16) 0.0265(6)$ Uani 11 d . . H12A H 0.56120 .18961 .10850 .032 Uiso 11 calc R. .

C13A C $0.4027(3) \quad 0.21749(18) \quad 1.03720(17) \quad 0.0318(7)$ Uani 11 d . . H13A H $0.3130 \quad 0.20161 .07240 .038$ Uiso 11 calc R . .

C14A C $0.3887(3) \quad 0.24840(18) \quad 0.96428(16) \quad 0.0293(6)$ Uani $11 d$. . H14A H $0.28930 .2552 \quad 0.94890 .035$ Uiso 1 l calc R. .

C15A C $0.5238(3) \quad 0.26931(15) \quad 0.91398(15) \quad 0.0206(5)$ Uani 11 d . . C16A C $0.5148(3) \quad 0.29775(17) \quad 0.83290(15) \quad 0.0244(6)$ Uani 11 d . . H16A H $0.5466 \quad 0.25090 .7998 \quad 0.029$ Uiso 1 l calc R. . H16B H $0.4037 \quad 0.3197 \quad 0.8306 \quad 0.029$ Uiso 11 calc R. . C17A C $0.7416(3) \quad 0.35176(16) \quad 0.74565(14) \quad 0.0207(5)$ Uani 11 d . . H17A H 0.78420 .30240 .72150 .025 Uiso 11 calc $R$.

C19A C $0.7087(3) \quad 0.47865(16) \quad 0.77584(14) 0.0214(5)$ Uani 11 d . . C18A C $0.5931(3) \quad 0.43973(16) \quad 0.82455(15) \quad 0.0233(6)$ Uani $11 d$. C23A C $0.4842(4) \quad 0.47995(19) \quad 0.88210(17) \quad 0.0340(7)$ Uani 11 d . . H23A H $0.40490 .45350 .9156 \quad 0.041$ Uiso 1 l calc R. .

C22A C $0.4971(4) \quad 0.5603(2) \quad 0.88809(19) \quad 0.0424$ (8) Uani 11 d . . H22A H 0.42590 .58970 .92750 .051 Uiso 1 calc R . .

C21A C $0.6110(4) \quad 0.59991(18) \quad 0.83827(19) \quad 0.0379(8)$ Uani 11 d. . H21A H $0.6137 \quad 0.6557 \quad 0.8443 \quad 0.046$ Uiso 11 calc R . .

C20A C $0.7202(3) \quad 0.56075(17) \quad 0.78044(16) 0.0283(6)$ Uani 11 d . . H20A H $0.79760 .5877 \quad 0.7462 \quad 0.034$ Uiso 1 l calc R. .

C24A C $0.9422(3) \quad 0.43630(17) \quad 0.66993(16) 0.0270(6)$ Uani 11 d. . H24A H 1.02620 .44450 .69720 .032 Uiso 11 calc R. . H24B H $0.9180 \quad 0.4867 \quad 0.63890 .032$ Uiso 1 l calc R. . C25A C $1.0030(4) 0.3678(2) \quad 0.61736(19) \quad 0.0411(8) \quad U a n i 11$. . H25A H 1.03140 .31810 .64750 .062 Uiso 1 l calc R . H25B H 1.09690 .38110 .58020 .062 Uiso 11 calc $\mathrm{R}$. . $\mathrm{H} 25 \mathrm{C} \mathrm{H} 0.92040 .35950 .59010 .062$ Uiso 1 l calc $\mathrm{R}$. . N3A N $0.6652(3) \quad 0.26238(13) \quad 0.93396(12) 0.0202(4)$ Uani 11 d . N2A N $0.9547(2) \quad 0.16651(13) \quad 0.97848(12) 0.0184(4)$ Uani 11 . . N1A N $1.1045(2) \quad 0.10352(12) 0.88131(11) 0.0177(4)$ Uani 11 d. . N4A N $0.6166(3) \quad 0.36027(13) \quad 0.80302$ (12) 0.0216 (5) Uani 11 d . .

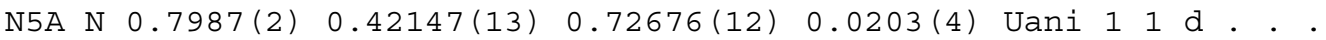
C1B C $0.9390(3) \quad 0.16047(14) \quad 0.40468$ (14) 0.0175 (5) Uani 11 d . . $\begin{array}{llllllllll}\mathrm{H} 1 \mathrm{~B} & \mathrm{H} & 0.8698 & 0.1947 & 0.3773 & 0.021 & \mathrm{UisO} & 1 & 1 & \mathrm{CalC} \\ \mathrm{R}\end{array}$.

C2B C $1.1392(3) \quad 0.07237(14) \quad 0.43014(14) 0.0184(5)$ Uani 11 d. . C3B C $1.0494(3) \quad 0.10143(14) 0.49916(14) 0.0173$ (5) Uani $11 d$. . C4B C $1.0880(3) \quad 0.07367(15) \quad 0.56932$ (15) 0.0198 (5) Uani 11 d. . H4B H 1.02660 .09300 .61640 .024 Uiso 11 calc $\mathrm{R}$. . C5B C $1.2213(3) \quad 0.01604(16) 0.56660(16) 0.0246(6)$ Uani 11 d . H5B H $1.2524-0.00460 .61320 .030$ Uiso 1 calc R. .

C6B C $1.3106(3)-0.01228(16) 0.49763(17) 0.0261(6)$ Uani 11 d . . H6B H $1.4014-0.05150 .49840 .031$ Uiso 1 l calc R. .

C7B C $1.2718(3) \quad 0.01464(15) \quad 0.42811(16) 0.0235$ (6) Uani 11 d . . H7B H $1.3329-0.00530 .38120 .028$ Uiso 11 calc $\mathrm{R}$. .

C8B C $1.1158(3) \quad 0.09826(17) \quad 0.29019(14) 0.0224$ (5) Uani 11 d . . H8B1 H 1.02550 .11570 .26420 .027 Uiso 1 l calc R . . H8B2 H $1.1508 \quad 0.0398 \quad 0.28220 .027$ Uiso 11 calc R. . C9B C $1.2509(4) \quad 0.1460(2) \quad 0.25487(17) \quad 0.0386(8)$ Uani 11 d. . . H9B1 H $1.21630 .2038 \quad 0.2626 \quad 0.058$ Uiso 11 calc $R$. H9B2 H $1.28190 .1373 \quad 0.19980 .058$ Uiso 1 l calc $R$. . H9B3 H $1.3416 \quad 0.1276 \quad 0.27940 .058$ Uiso 1 l calc R . . C10B C $0.8119(3) \quad 0.20904(15) \quad 0.53477(14) \quad 0.0182$ (5) Uani 11 d . . H10C H $0.8418 \quad 0.26450 .52650 .022$ Uiso 1 l calc R. . H1OD H $0.82210 .1894 \quad 0.5876 \quad 0.022$ Uiso 1 calc R . . C11B C $0.6413(3) \quad 0.21324(14) 0.52835(14) 0.0183(5)$ Uani 11 d . . C12B C $0.5262(3) \quad 0.19751$ (15) 0.59227 (15) $0.0217(5)$ Uani 11 d . $\begin{array}{lllllllllll}\mathrm{H} 12 \mathrm{~B} & \mathrm{H} & 0.5544 & 0.1797 & 0.6401 & 0.026 & \text { Uiso } 1 & 1 & \mathrm{CalC} & \mathrm{R}\end{array}$.

C13B C $0.3697(3) \quad 0.20834(16) \quad 0.58474(15) \quad 0.0249(6)$ Uani 11 d . . H13B H $0.28810 .1983 \quad 0.6275 \quad 0.030$ Uiso 1 l calc R. .

C14B C $0.3332(3) \quad 0.23403(15) \quad 0.51409(15) \quad 0.0231$ (5) Uani 11 d . . H14B H $0.22650 .24190 .5073 \quad 0.028$ Uiso 1 l calc R. . 
C15B C $0.4556(3) \quad 0.24796(14) \quad 0.45371(14) \quad 0.0179(5)$ Uani 11 d . . C16B C $0.4245(3) 0.27661(15) \quad 0.37493(14) 0.0200(5)$ Uani 11 d . . $\mathrm{H} 16 \mathrm{C} \mathrm{H} 0.4597 \quad 0.23170 .3397 \quad 0.024$ Uiso 1 l calc $\mathrm{R}$. . H16D H $0.3093 \quad 0.29290 .3781 \quad 0.024$ Uiso 11 calc R . . C17B C $0.6386(3) \quad 0.34209(16) \quad 0.29024(15) \quad 0.0211(5)$ Uani 11 d... H17B H $0.6868 \quad 0.2952 \quad 0.2632 \quad 0.025$ Uiso 1 l calc R. . C18B C $0.4767(3) \quad 0.42315(15) \quad 0.37291(14) \quad 0.0193(5)$ Uani 11 d . C19B C $0.5930(3) \quad 0.46619(16) \quad 0.32994(15) \quad 0.0212(5)$ Uani $11 d$. C20B C $0.5969(3) \quad 0.54721(16) \quad 0.34238(17) \quad 0.0276(6)$ Uani 11 d . . H2OB H $0.6746 \quad 0.5775 \quad 0.3127 \quad 0.033$ Uiso 1 1 Calc R. . C21B C $0.4804(4) \quad 0.58041(17) \quad 0.40055(18) 0.0323(7)$ Uani 11 d. . H21B H $0.47990 .6351 \quad 0.41180 .039$ Uiso 11 calc $\mathrm{R}$. . C22B C $0.3623(4) \quad 0.53706(17) \quad 0.44399(17) 0.0310(6)$ Uani 11 d . . H22B H $0.28460 .5630 \quad 0.48340 .037$ Uiso 1 1 calc R . C23B C $0.3578(3) \quad 0.45731(17) \quad 0.43019(16) 0.0250(6)$ Uani 11 d. . H23B H $0.2776 \quad 0.4276 \quad 0.45840 .030$ Uiso 1 l calc R. . C24B C $0.8343(3) \quad 0.43321(17) \quad 0.22421(17) 0.0291(6)$ Uani 11 d. . H24C H $0.9120 \quad 0.44590 .25330 .035$ Uiso 11 calc $\mathrm{R}$. .

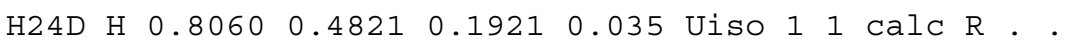
C25B C $0.9100(4) \quad 0.3645(2) \quad 0.17299(19) \quad 0.0425$ (8) Uani 11 d . . $\mathrm{H} 25 \mathrm{D} \mathrm{H} 0.94420 .31690 .20440 .064$ Uiso 11 calc $\mathrm{R}$. . H25E H $1.0020 \quad 0.38110 .13650 .064$ Uiso 11 calc R. . $\mathrm{H} 25 \mathrm{~F} \mathrm{H} 0.83290 .35090 .1448 \quad 0.064$ Uiso 11 calc R. . N3B N $0.6076(2) \quad 0.23795(12) 0.45983(12) 0.0174(4)$ Uani 11 d . N2B N $0.9239(2) \quad 0.15622(12) \quad 0.48051(11) \quad 0.0166(4) \quad \operatorname{Uani} 11 d$. N1B N $1.0640(2) \quad 0.11042(13) \quad 0.37248(12) 0.0189(4)$ Uani $11 d .$. N4B N $0.5092(3) \quad 0.34523(12) \quad 0.34507(12) 0.0190(4) \quad \operatorname{Uani} 11 d$. N5B N $0.6912(3) \quad 0.41338(13) \quad 0.27839(12) 0.0213$ (5) Uani 11 . . Br1 Br $0.21709(3) \quad 0.865324(16) \quad 0.258850(15) \quad 0.02442(7)$ Uani $11 d . .$. Br2 Br $0.71260(3) \quad 0.141088(16) \quad 0.238593(15) 0.02486(7)$ Uani $11 d$. Br3 Br $-0.00467(3) \quad 0.351154(16) 0.393582(16) 0.02643(7)$ Uani $11 d$. Br4 Br $0.09025(16) 0.34521(10) 0.83815(16) 0.0487(6)$ Uani $0.412(3) 1$ d P.

Br4A Br $0.12647(9) \quad 0.31787(6) \quad 0.79565(8) \quad 0.0341(3)$ Uani $0.588(3) \quad 1$ d P . C60 C $-0.1271(6) 0.4476(4) 0.9618(4) 0.106(2)$ Uani 11 d . .

H60A H -0.21720 .41690 .97750 .159 Uiso 11 calc R. .

H60B H -0.12640 .47190 .90990 .159 Uiso 11 calc $R$. .

H60C H -0.13680 .49050 .99750 .159 Uiso 11 calc R .

O $00.0231(6) \quad 0.3921(3) \quad 0.9627(3) \quad 0.1347(17)$ Uani 11 d . .

H1O H 0.06750 .38110 .91750 .202 Uiso 11 calc R. .

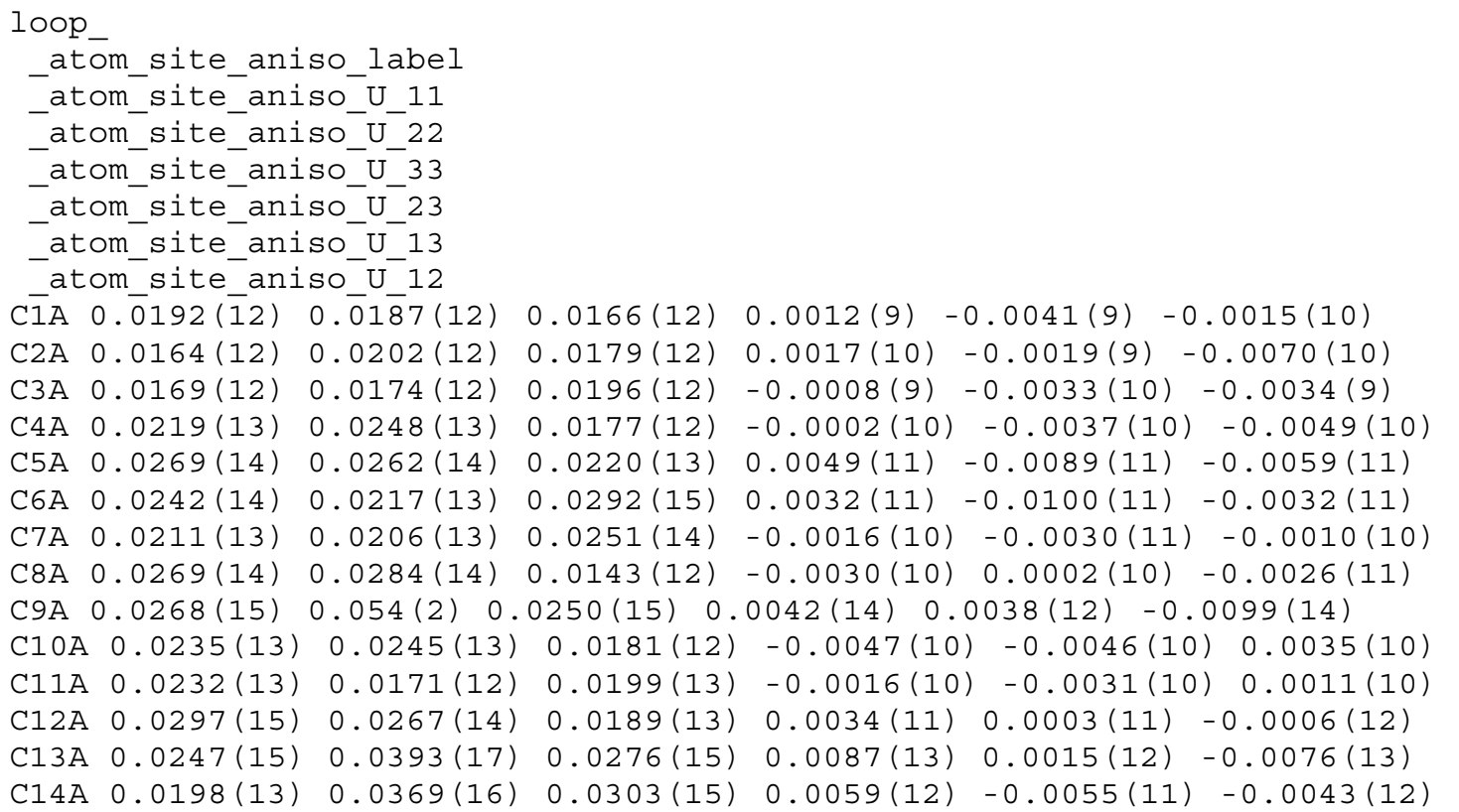




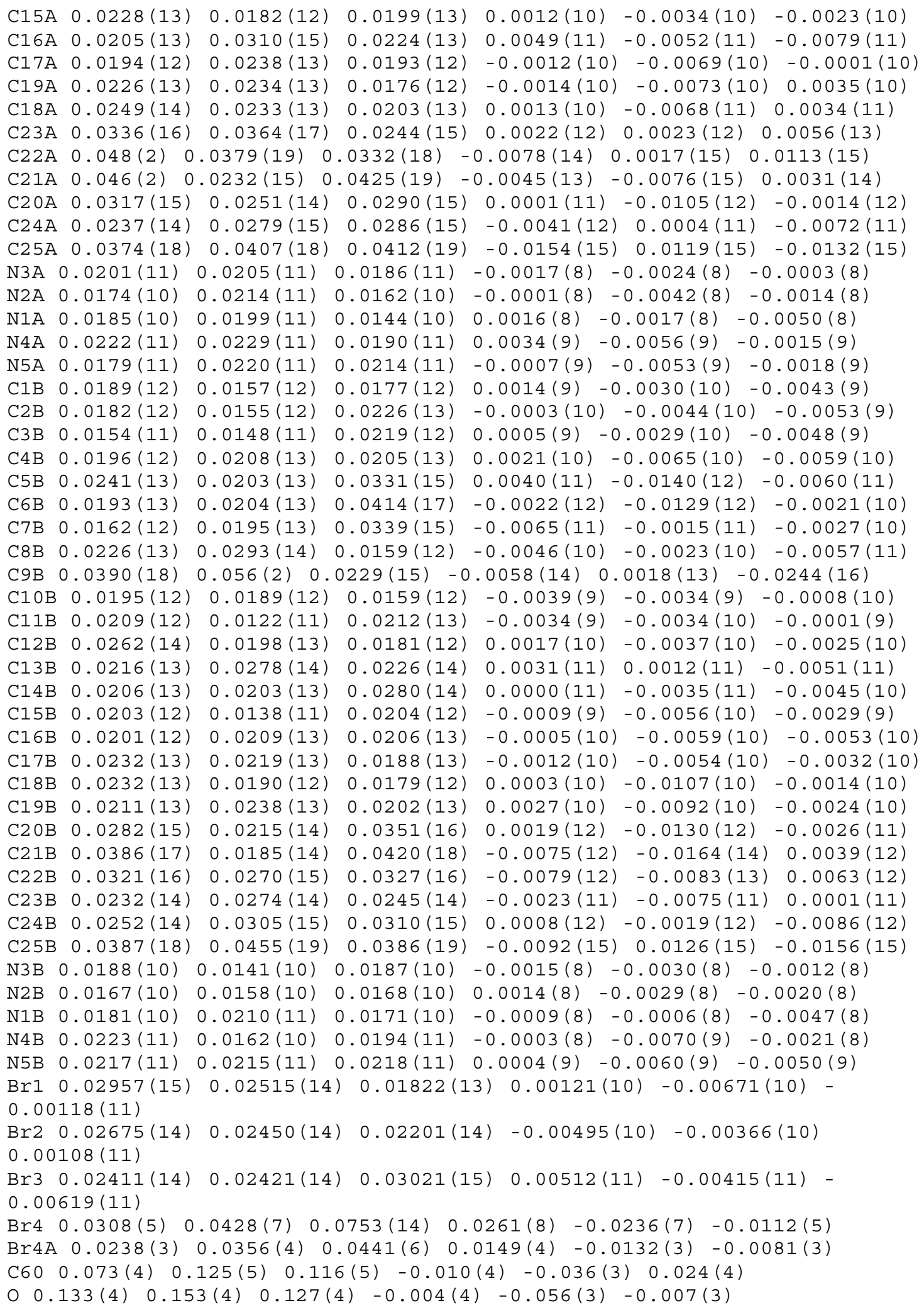




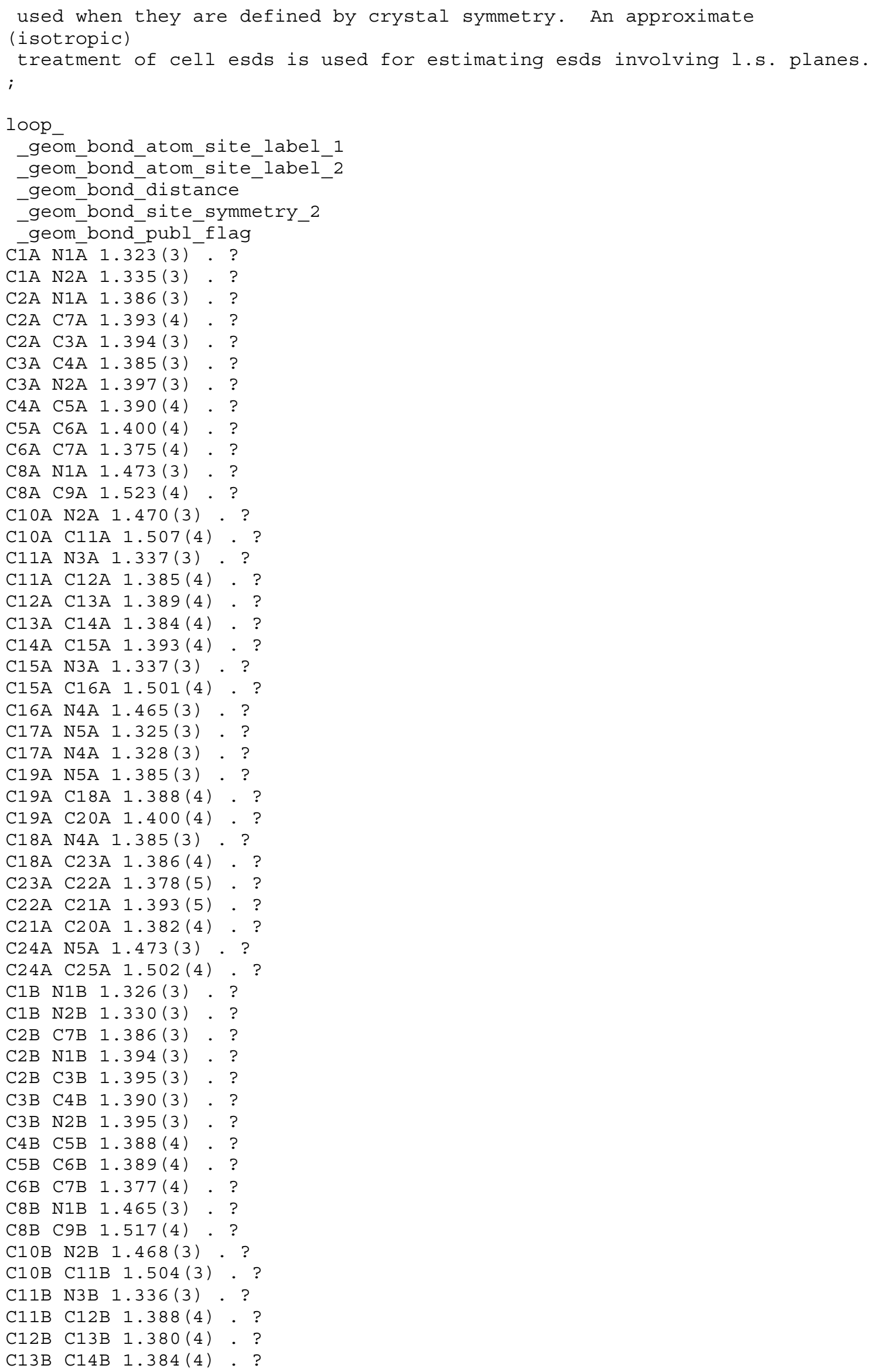




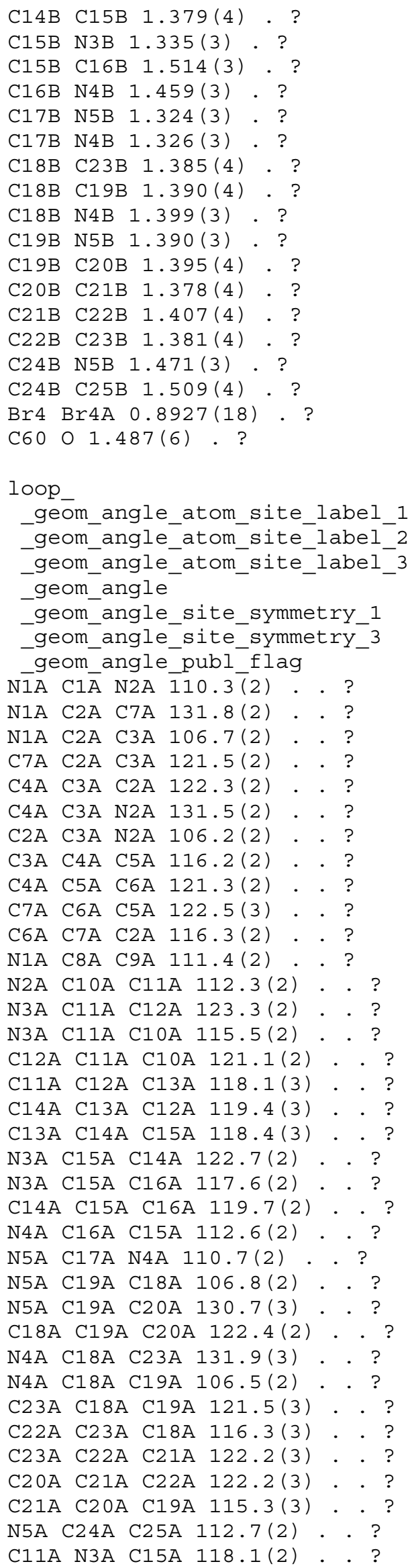




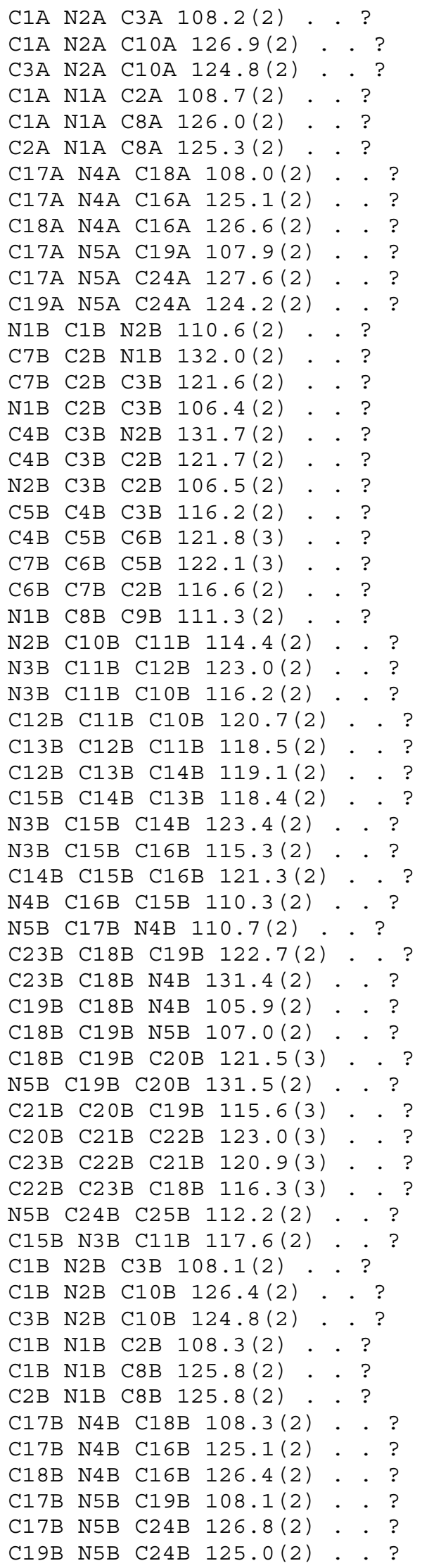


diffrn measured fraction theta full

-refine diff density max - $0.9 \overline{2} 7$

0.982

refine_diff_density_min -0.755

_refine_diff_density_rms 0.095 


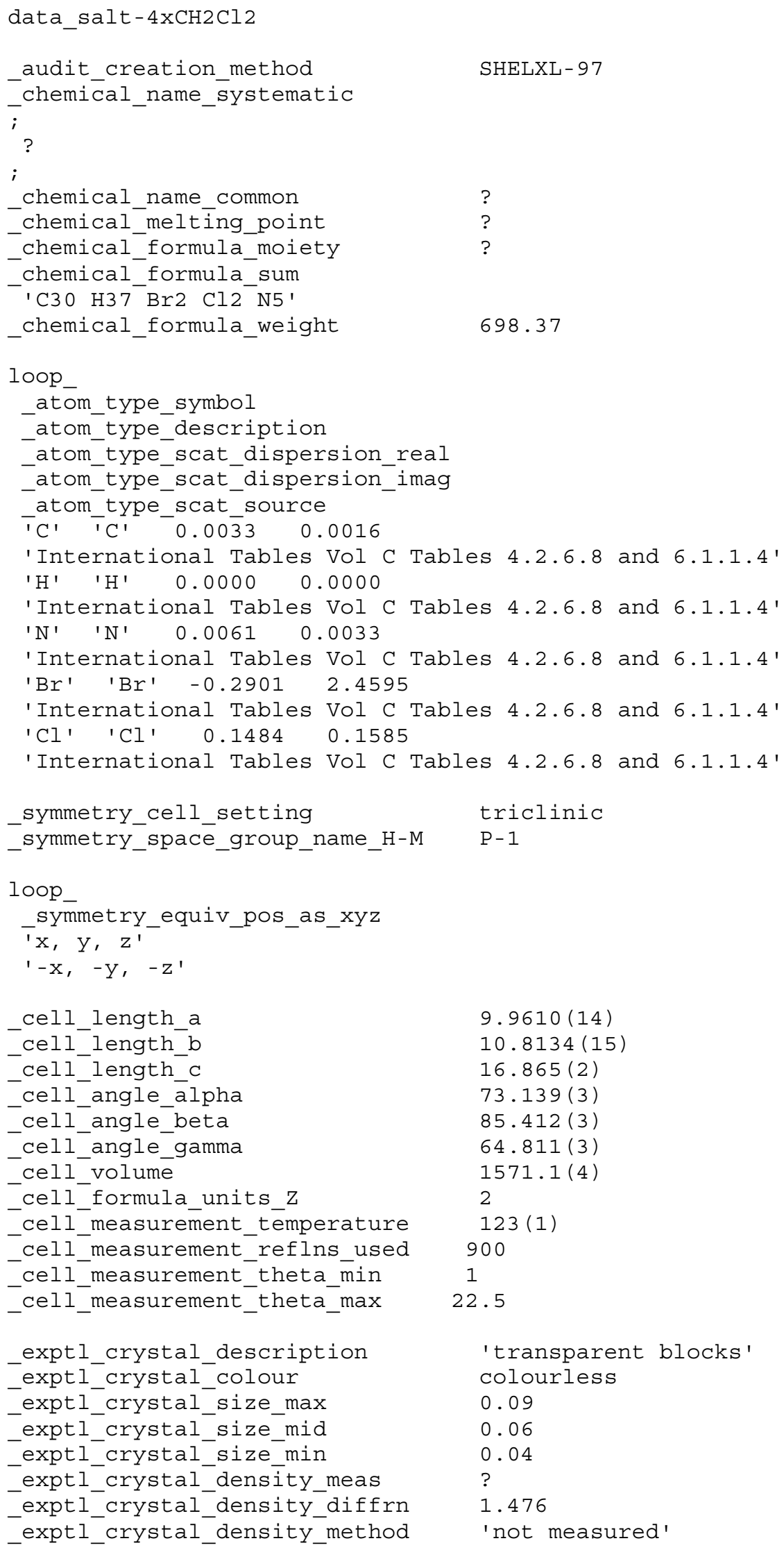




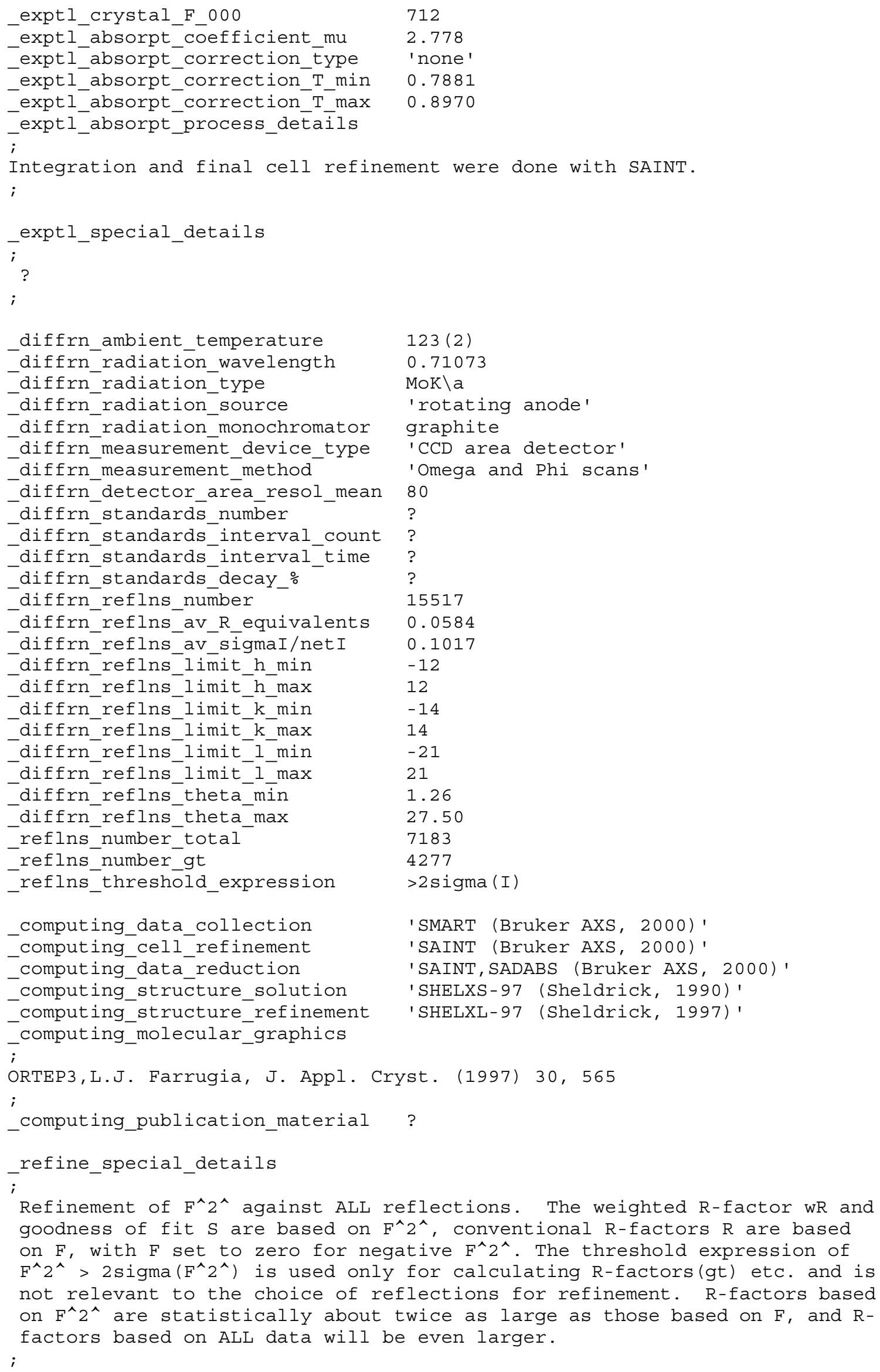




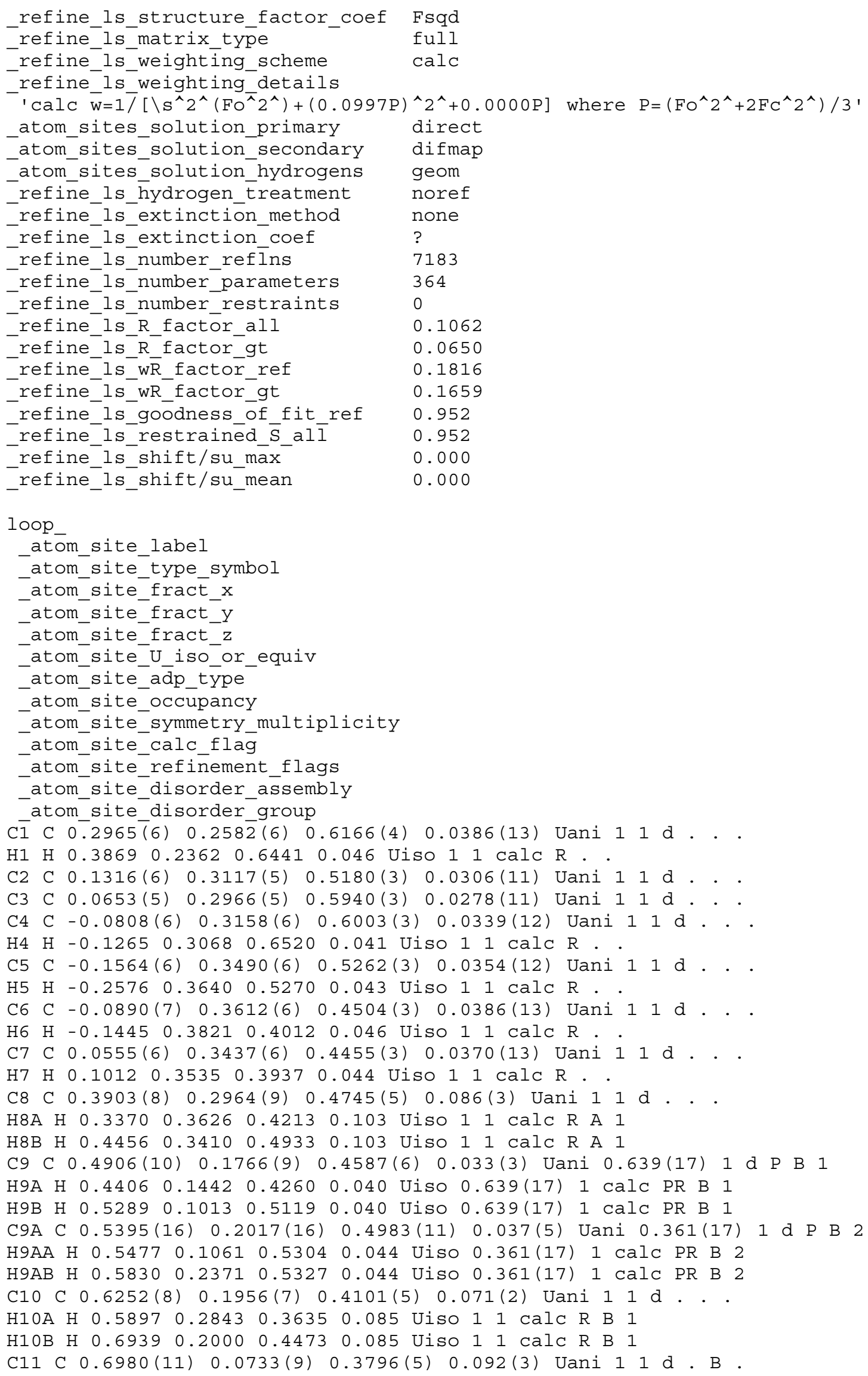


H11A H $0.7401-0.0127 \quad 0.42650 .137$ Uiso 11 calc $R$. .

H11B H $0.7778 \quad 0.0836 \quad 0.3443 \quad 0.137$ Uiso 11 calc R. . H11C H $0.6260 \quad 0.0655 \quad 0.3473 \quad 0.137$ Uiso 11 calc R . . C12 C $0.1514(6) \quad 0.2436(6) \quad 0.7430(3) \quad 0.0366$ (13) Uani 11 d . . $\begin{array}{llllllllllll}\mathrm{H} 12 \mathrm{~A} & \mathrm{H} & 0.2491 & 0.2016 & 0.7731 & 0.044 & \text { Uiso } 1 & 1 & \mathrm{calC} & \mathrm{R}\end{array}$. $\mathrm{H} 12 \mathrm{~B} \mathrm{H} 0.1046 \quad 0.1764 \quad 0.76350 .044$ Uiso 1 l calc R. . C13 C $0.0546(5) \quad 0.3834(5) \quad 0.7601(3) \quad 0.0289$ (11) Uani 11 d . . C14 C $0.1124(6) \quad 0.4781(6) 0.7603(3) \quad 0.0319(12)$ Uani 11 d. . $\mathrm{H} 14 \mathrm{H} \quad 0.2145 \quad 0.4557 \quad 0.7512 \quad 0.038$ Uiso 11 calc R. . C15 C $0.0178(6) \quad 0.6080(6) 0.7742(3) \quad 0.0342(12)$ Uani 11 d . . $\mathrm{H} 15 \mathrm{H} \quad 0.05440 .6756 \quad 0.7753 \quad 0.041$ Uiso 11 calc $\mathrm{R}$.

C16 C $-0.1301(6) \quad 0.6367(6) \quad 0.7864(3) \quad 0.0308(11)$ Uani 11 d. . $\mathrm{H} 16 \mathrm{H}-0.19770 .72470 .79540 .037$ Uiso 11 calc $\mathrm{R}$. .

C17 C $-0.1767(5) \quad 0.5337(5) \quad 0.7853$ (3) 0.0273 (11) Uani 11 d . . C18 C $-0.3348(5) \quad 0.5547(5) \quad 0.8015(3) \quad 0.0300(11)$ Uani 11 d. . H18A H -0.39270 .64920 .81090 .036 Uiso 11 calc R. . $\mathrm{H} 18 \mathrm{~B}$ H $-0.3807 \quad 0.55180 .75250 .036$ Uiso 11 calc $\mathrm{R}$. . C19 C $-0.3458(6) \quad 0.3245(6) \quad 0.8739(4) \quad 0.0359(12)$ Uani 11 d . . $\mathrm{H} 19 \mathrm{H}-0.35550 .3004 \quad 0.8254 \quad 0.043$ Uiso 11 Calc R. . C20 C $-0.3236(5) \quad 0.3127(6) \quad 1.0052(3) \quad 0.0327(12)$ Uani 11 d . . C21 C $-0.3240(5) \quad 0.4413(5) \quad 0.9564(3) \quad 0.0299(11)$ Uani 11 d . . C22 C $-0.3162(5) \quad 0.5418(6) \quad 0.9894(3) \quad 0.0314(11)$ Uani 11 d . . $\mathrm{H} 22 \mathrm{H}-0.3170 \quad 0.62930 .9556 \quad 0.038$ Uiso 11 Calc R. .

C23 C $-0.3074(6) 0.5050(7) \quad 1.0748(4) \quad 0.0407(14)$ Uani 11 d . . $\mathrm{H} 23 \mathrm{H}-0.30090 .5691 \quad 1.10110 .049$ Uiso 11 calc R. .

C24 C $-0.3076(6) \quad 0.3755(7) \quad 1.1242(3) \quad 0.0408(14)$ Uani 11 d. . $\mathrm{H} 24 \mathrm{H}-0.30290 .35601 .18280 .049$ Uiso 11 calc $\mathrm{R}$. .

C25 C $-0.3143(6) \quad 0.2758(6) 1.0910(4) \quad 0.0398$ (13) Uani 11 d . . $\mathrm{H} 25 \mathrm{H}-0.31270 .18781 .12460 .048$ Uiso 11 calc $\mathrm{R}$. . C26 C $-0.3477(6) 0.1042(6) 0.9726(4) \quad 0.0440(14)$ Uani 11 d . . $\mathrm{H} 26 \mathrm{~A} \mathrm{H}-0.39620 .0990 \quad 0.92540 .053$ Uiso 11 calc $\mathrm{R}$. . $\mathrm{H} 26 \mathrm{~B} \mathrm{H}-0.41020 .09741 .02090 .053$ Uiso 11 calc R. .

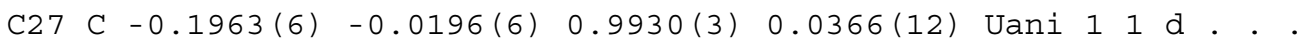
H27A H $-0.1501-0.01781 .04230 .044$ Uiso 11 calc R. . H27B H $-0.1319-0.01020 .94590 .044$ Uiso 11 calc $R$. . C28 C $-0.2079(6)-0.1606(6) 1.0104(4) \quad 0.0397(13)$ Uani $11 d$. . H28A H $-0.2564-0.17801 .06340 .048$ Uiso 11 calc $R$. H28B H $-0.2709-0.15510 .96580 .048$ Uiso 11 calc $R$. C29 C $-0.0576(6)-0.2832(6) 1.0153(4) \quad 0.0453(14)$ Uani $11 d$. . H29A H $-0.0126-0.27010 .96140 .068$ Uiso 11 calc R. . H29B H $-0.0690-0.37271 .02970 .068$ Uiso 11 calc $R$. $\mathrm{H} 29 \mathrm{C}$ H $0.0064-0.2867 \quad 1.0578 \quad 0.068$ Uiso 11 calc R. . N1 N $0.2769(5) \quad 0.2874(5) \quad 0.5358(3) \quad 0.0398(11)$ Uani 11 d. B . $\mathrm{N} 2 \mathrm{~N} \quad 0.1727(4) \quad 0.2634(4) \quad 0.6544(3) \quad 0.0303(10) \quad \operatorname{Uani} 11 \mathrm{~d} . \quad$. N3 N $-0.0863(4) \quad 0.4090(4) \quad 0.7726(2) \quad 0.0287(9)$ Uani 11 d . . $\mathrm{N} 4 \mathrm{~N}-0.3384(5) \quad 0.4435(4) \quad 0.8738(3) \quad 0.0304(9) \quad \operatorname{Uani} 11 \mathrm{~d} .$. N5 N - 0.3377(5) $0.2432(5) 0.9508(3) \quad 0.0366(11)$ Uani $11 d . .$. C99 C $0.7961(7) \quad 0.0412(7) \quad 0.6964(4) 0.0541(17)$ Uani 11 d. . H99A H $0.7486 \quad 0.14620 .67340 .065$ Uiso 1 l calc R. . H99B H $0.7187 \quad 0.0100 \quad 0.7222 \quad 0.065$ Uiso 11 calc R. Cl1 Cl $0.9340(2) \quad-0.0068(2) \quad 0.77193(11) 0.0721(5)$ Uani 11 d . . Cl2 Cl $0.8713(3) \quad-0.0369(2) \quad 0.61548(13) 0.0856(7) \quad \operatorname{Uani} 11 \mathrm{~d}$. Br1 Br $0.49041(6) \quad 0.59221(6) \quad 0.32884(3) \quad 0.03711(18)$ Uani $11 d$. . Br2 Br $0.48562(6) 0.05201(6) \quad 0.18612(4) \quad 0.0504(2)$ Uani 11 d. .

\footnotetext{
loop

atom_site_aniso_label

_atom_site_aniso_U_11

atom site aniso U 22

atom site aniso U 33

atom_site_aniso_U_23

atom_site_aniso_U_13

atom_site_aniso_U_12
} 


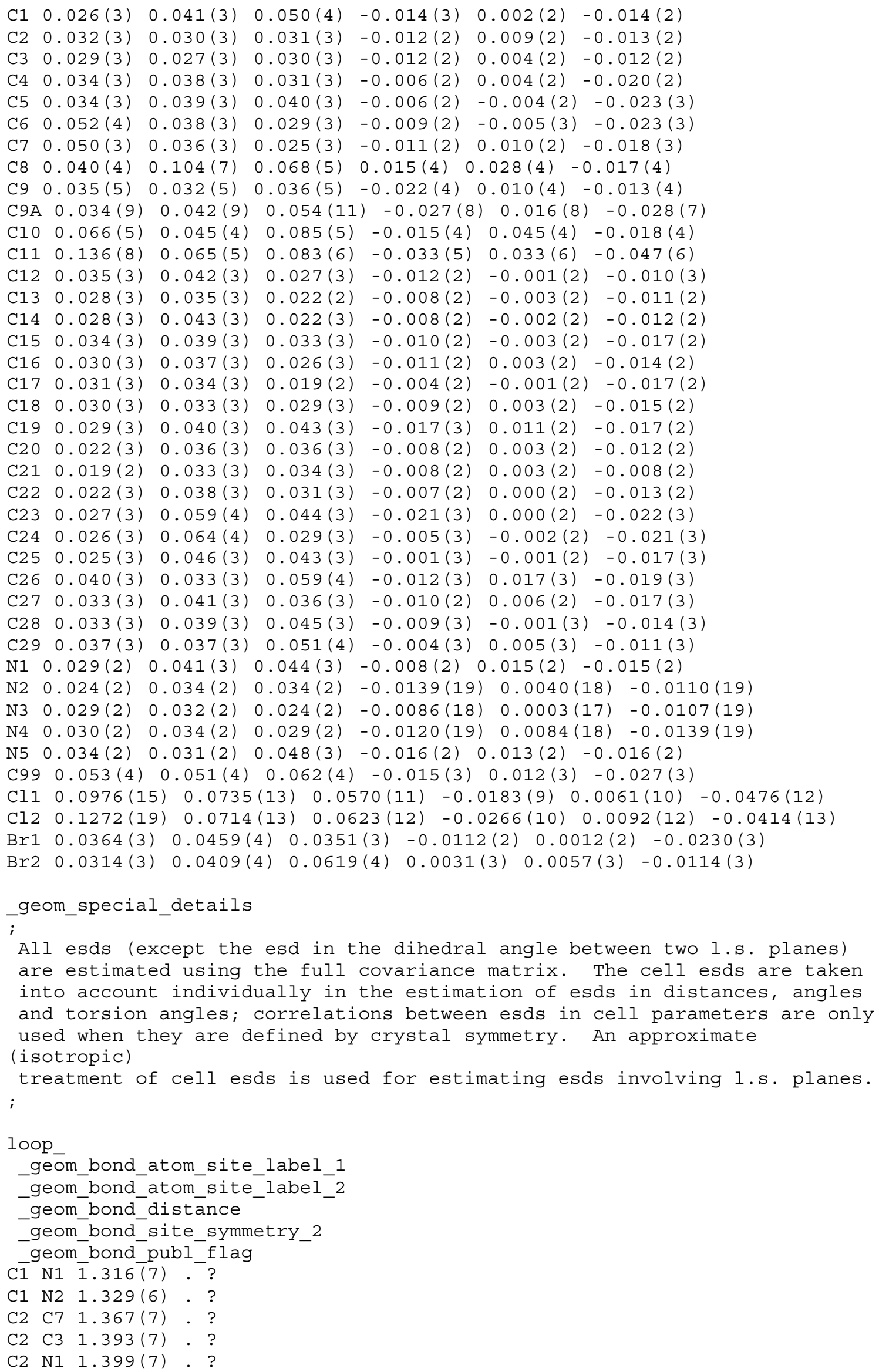




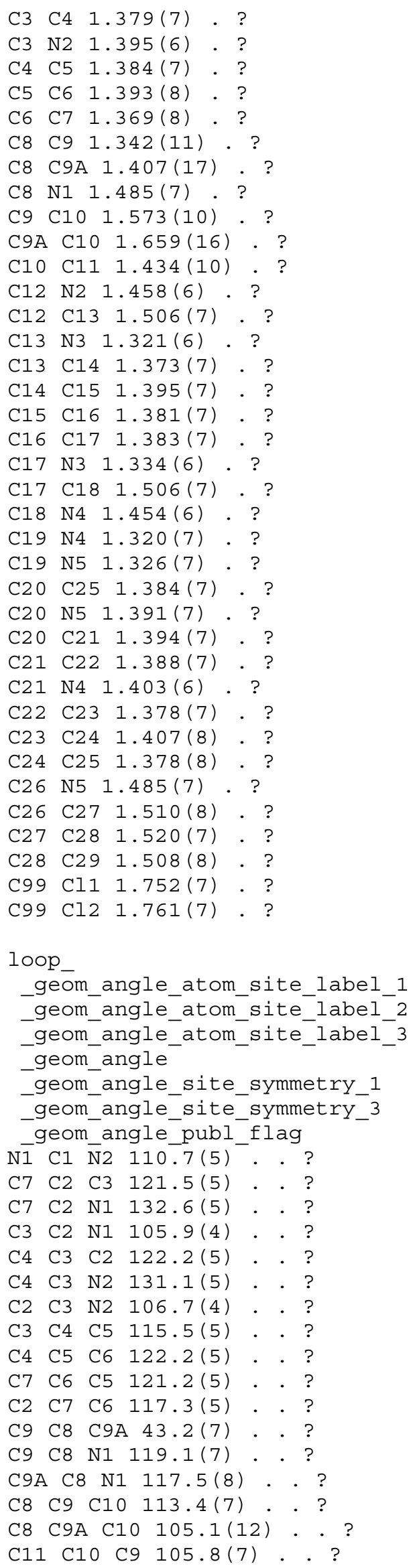




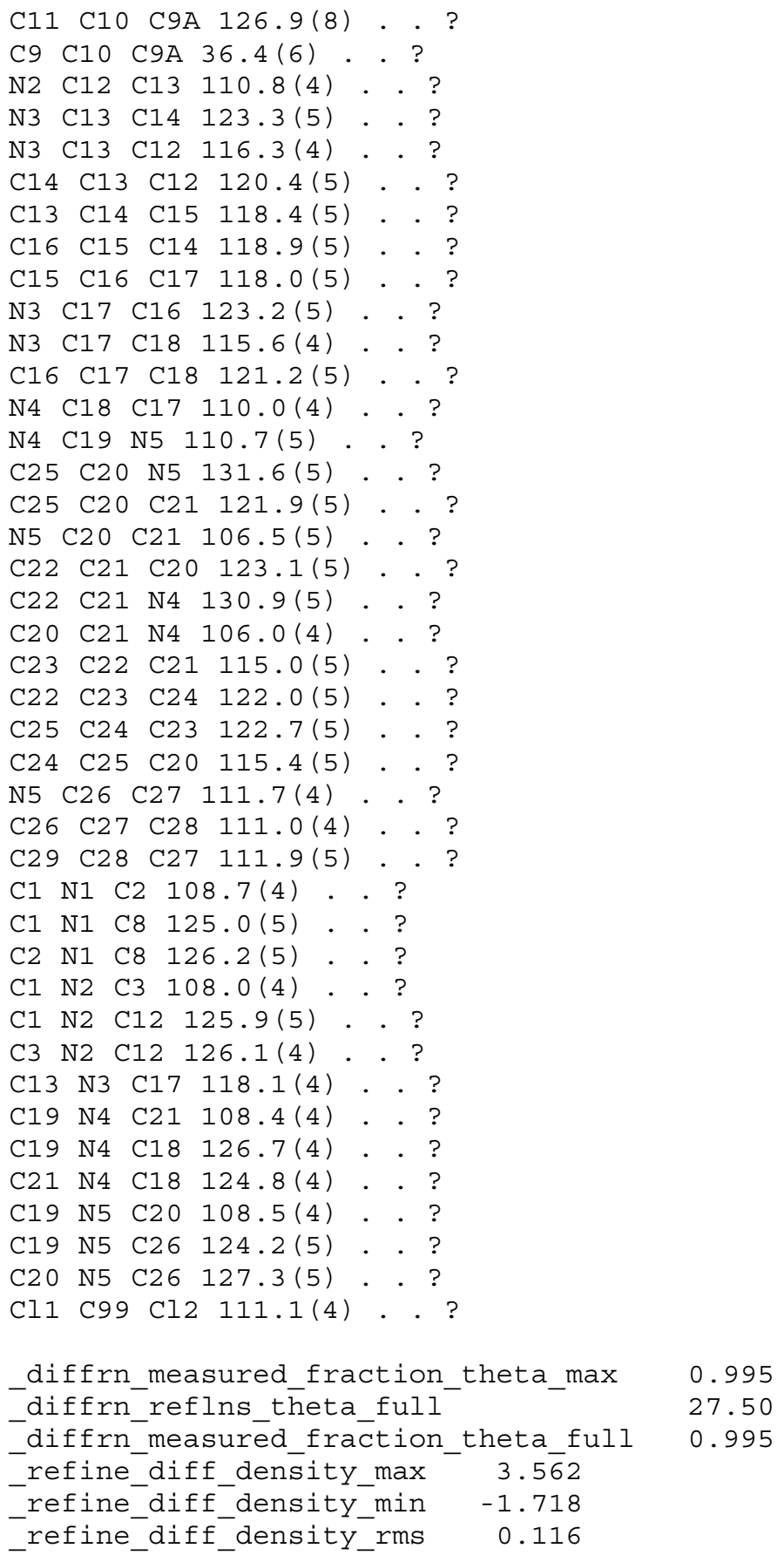




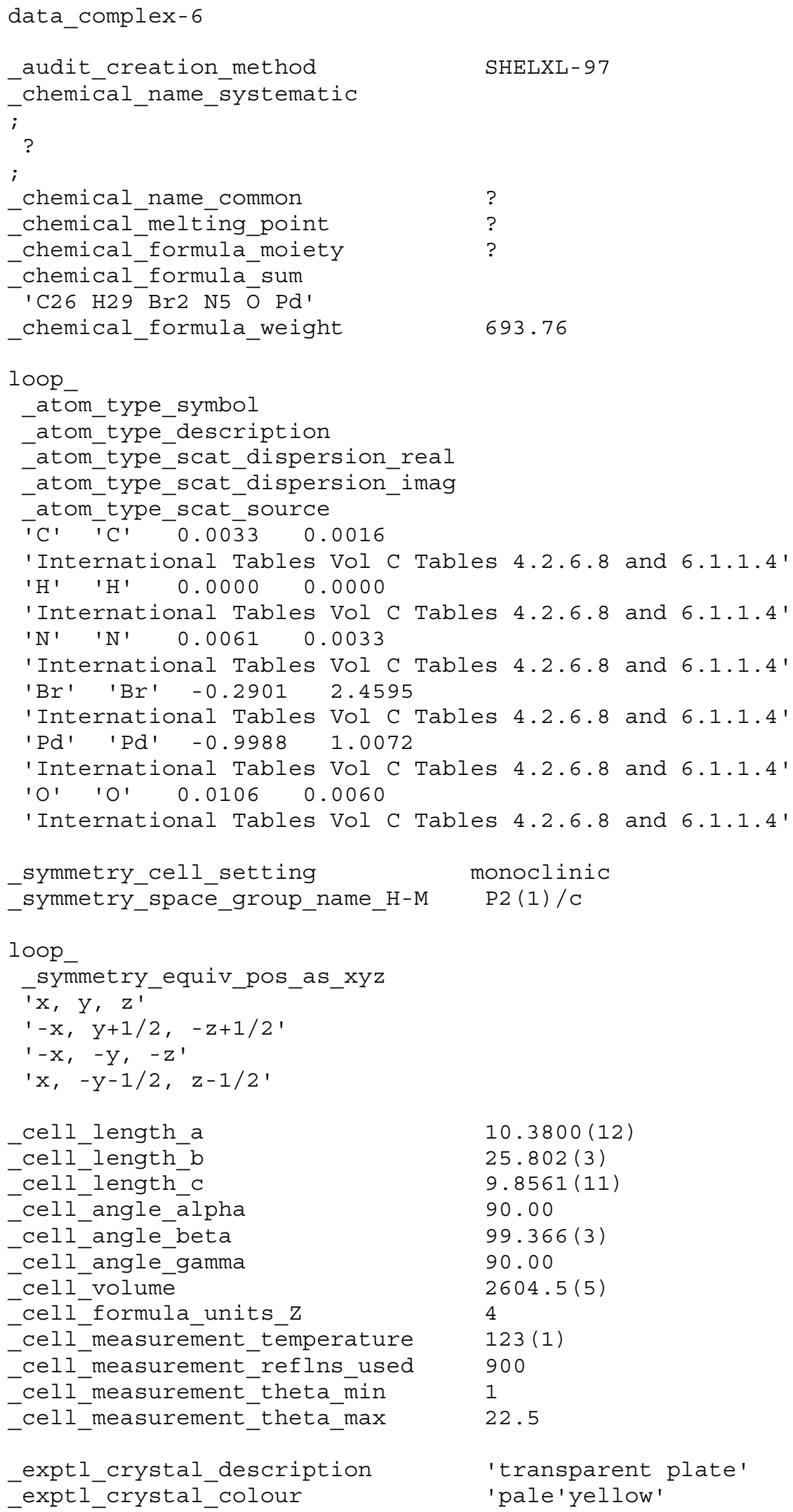




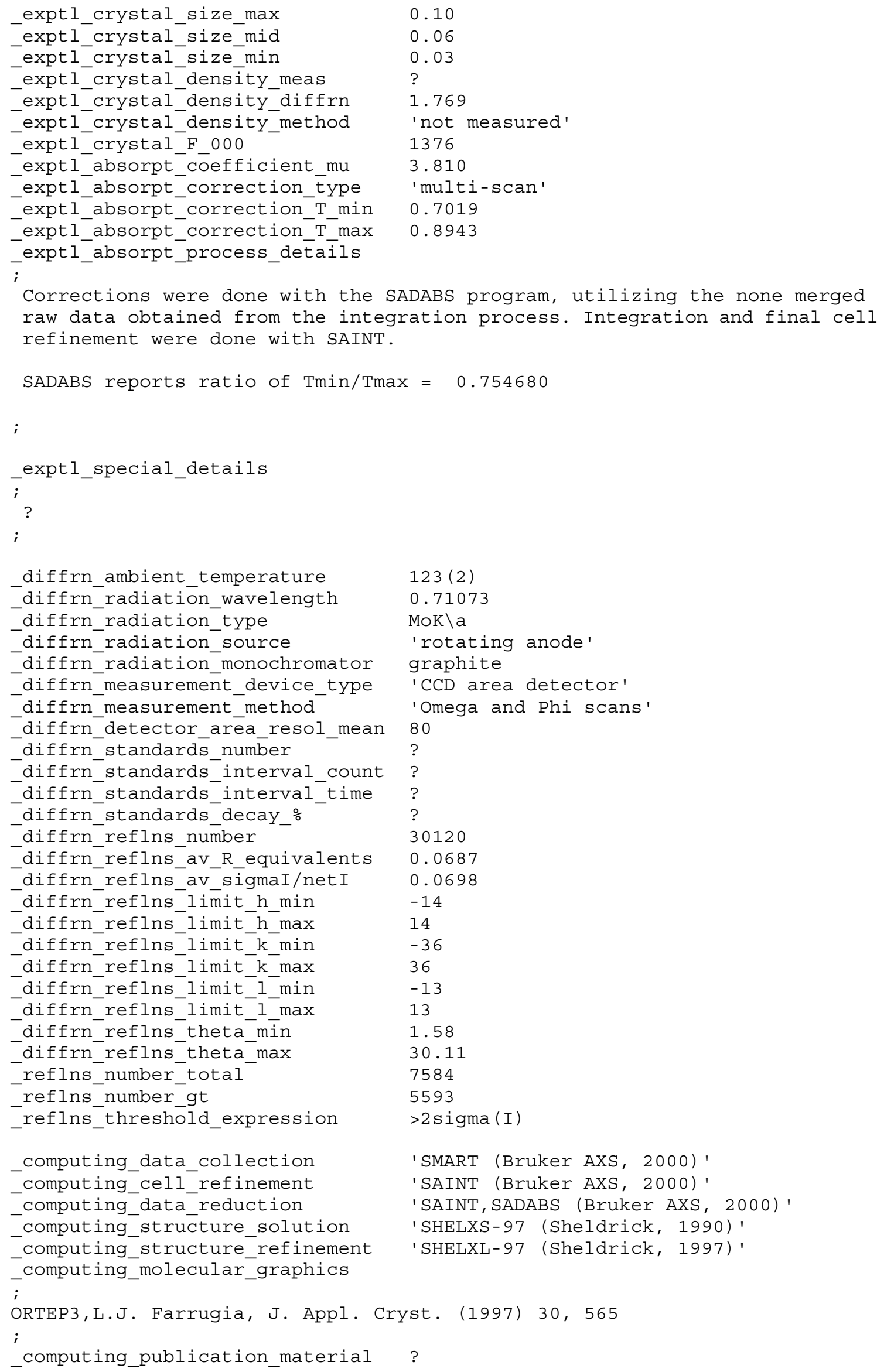




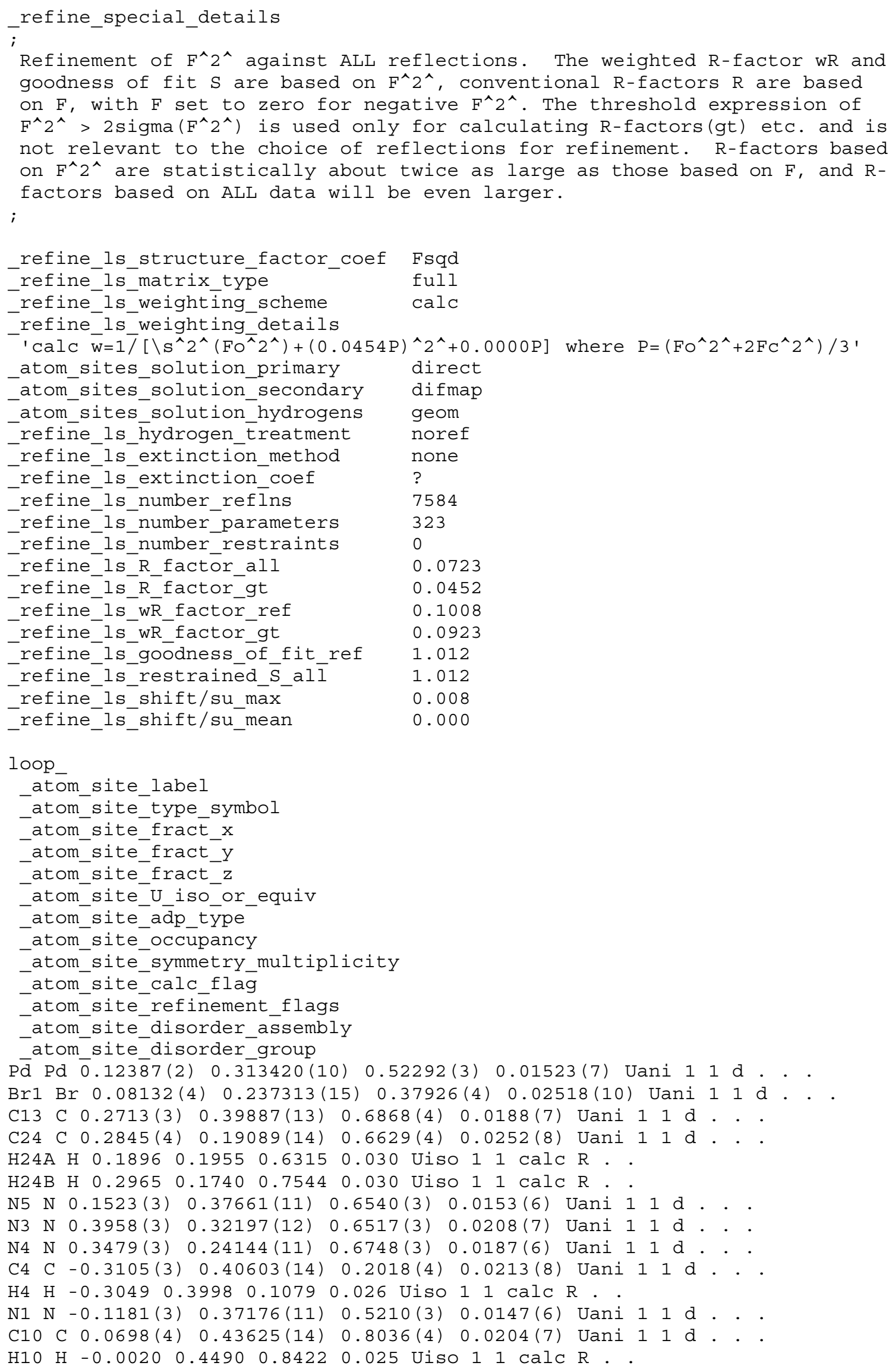


C15 C $0.2998(3) \quad 0.28646(14) 0.6195(4) \quad 0.0183(7)$ Uani 11 d . . C1 C $-0.0448(3) \quad 0.34802(13) 0.4368(4) \quad 0.0178(7)$ Uani 11 d. . C8 C $-0.0794(3) \quad 0.37137(13) \quad 0.6703(3) \quad 0.0158(7)$ Uani 11 d . . H8A H -0.07750 .33520 .70410 .019 Uiso 1 l calc R. . H8B H $-0.14450 .3908 \quad 0.71330 .019$ Uiso 1 l calc $\mathrm{R}$. . C9 C $0.0527(3) \quad 0.39546(13) \quad 0.7114(3) \quad 0.0163(7) \quad \operatorname{Uani} 11$ d. . . C2 C $-0.2265(3) \quad 0.39625(13) 0.4455(3) \quad 0.0158(7)$ Uani 11 d . . C3 C $-0.2168(3) \quad 0.38719(14) 0.3081(4) \quad 0.0187(7)$ Uani 11 d. . C6 C $-0.4214(4) \quad 0.44296(14) \quad 0.3778(4) \quad 0.0238(8)$ Uani 11 d. . H6 H $-0.4934 \quad 0.4622 \quad 0.3994 \quad 0.029$ Uiso 11 calc $\mathrm{R}$. . C14 C $0.3782(3) \quad 0.37696(14) \quad 0.6182(4) \quad 0.0219(8)$ Uani 11 d. . H14A H $0.3556 \quad 0.38130 .5174 \quad 0.026$ Uiso 11 calc R . . H14B H $0.4607 \quad 0.39590 .64980 .026$ Uiso 1 l calc $\mathrm{R}$. . C7 C $-0.3289(3) \quad 0.42441(14) \quad 0.4839(4) \quad 0.0209(8)$ Uani 11 d. . $\mathrm{H} 7 \mathrm{H}-0.3351 \quad 0.4306 \quad 0.5776 \quad 0.025$ Uiso 11 calc R. .

$\mathrm{N} 2 \mathrm{~N}-0.1053(3) \quad 0.35760(12) \quad 0.3077(3) \quad 0.0185(6) \quad \operatorname{Uani} 11 \mathrm{~d}$. . C5 C $-0.4117(4) \quad 0.43415(14) \quad 0.2403(4) \quad 0.0230(8)$ Uani 11 d . . $\mathrm{H} 5 \mathrm{H}-0.47690 .44790 .17090 .028$ Uiso $11 \mathrm{calc} R$. .

C11 C $0.1925(4) \quad 0.45843(14) 0.8396(4) \quad 0.0235(8)$ Uani 11 d. .

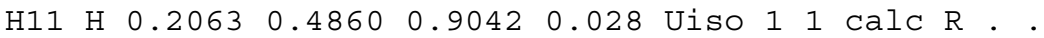

C17 C $0.6234(4) \quad 0.32141(16) \quad 0.7917(4) \quad 0.0288(9)$ Uani 11 d. . $\mathrm{H} 17 \mathrm{H} \quad 0.6440 \quad 0.35690 .7810 \quad 0.035$ Uiso 11 calc R. .

C16 C $0.5045(4) \quad 0.30036(15) \quad 0.7315(4) \quad 0.0225(8)$ Uani 11 d . . C21 C $0.4742(4) \quad 0.24864(15) \quad 0.7477(4) \quad 0.0241(8)$ Uani 11 d . . C12 C $0.2941(4) \quad 0.43941(14) \quad 0.7792(4) \quad 0.0242(8)$ Uani 11 d . . . H12 H $0.3788 \quad 0.4541 \quad 0.8010 \quad 0.029$ Uiso 1 l calc R. .

Br2 Br $0.69360(4) \quad 0.035677(15) \quad 0.34573(4) \quad 0.02496(10)$ Uani $11 d$. . $000.2293(3) \quad 0.44637(15) \quad 0.3684(4) \quad 0.0553(11)$ Uani 11 d . . . C22 C $-0.0626(4) \quad 0.34081(17) \quad 0.1810(4) 0.0295(9)$ Uani $11 d$. . H22A H -0.13780 .32560 .11920 .035 Uiso 11 calc R . $\mathrm{H} 22 \mathrm{~B} \mathrm{H} 0.00430 .31340 .2028 \quad 0.035$ Uiso 11 calc R. .

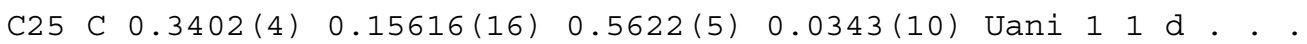
H25A H $0.32250 .17150 .4700 \quad 0.051$ Uiso 1 calc R. . H25B H 0.29930 .12190 .56050 .051 Uiso 11 calc $R$. $\mathrm{H} 25 \mathrm{C} \mathrm{H} \quad 0.43470 .1527 \quad 0.5910 \quad 0.051$ Uiso 1 1 calc R. . C20 C $0.5592(4) \quad 0.21500(16) \quad 0.8257(4) \quad 0.0324(10)$ Uani 11 d. . $\mathrm{H} 20 \mathrm{H} \quad 0.5376 \quad 0.1797 \quad 0.8374 \quad 0.039$ Uiso 11 calc $\mathrm{R}$. . C18 C $0.7096(4) \quad 0.28789(17) \quad 0.8677(4) \quad 0.0338(10)$ Uani 11 d . .

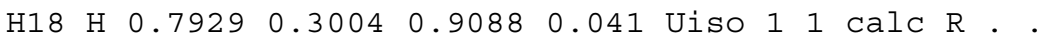
C19 C $0.6779(4) \quad 0.23576(17) \quad 0.8862$ (5) $0.0386(11)$ Uani 11 d. . H19 H 0.73940 .21420 .94150 .046 Uiso 11 calc R . . C23 C $-0.0072(5) \quad 0.38407(19) \quad 0.1076(5) \quad 0.0427$ (12) Uani 11 . . H23A H -0.07570 .40950 .07660 .064 Uiso 11 calc $\mathrm{R}$. . H23B H $0.02690 .3702 \quad 0.0278 \quad 0.064$ Uiso 1 l calc R. . H23C H 0.06370 .40080 .17010 .064 Uiso 1 l calc R. . C55 C $0.1210(5) \quad 0.47117(18) \quad 0.4084(5) \quad 0.0449(12)$ Uani 11 d. .

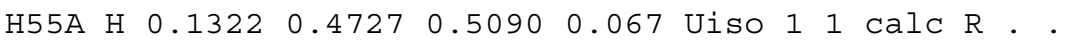
H55B H 0.04150 .45170 .37320 .067 Uiso 11 calc R . . H55C H 0.11370 .50640 .37090 .067 Uiso 1 l calc R . . H5O H $0.257(4) \quad 0.4637(16) \quad 0.309(4) \quad 0.020(11)$ Uiso 11 d . . .

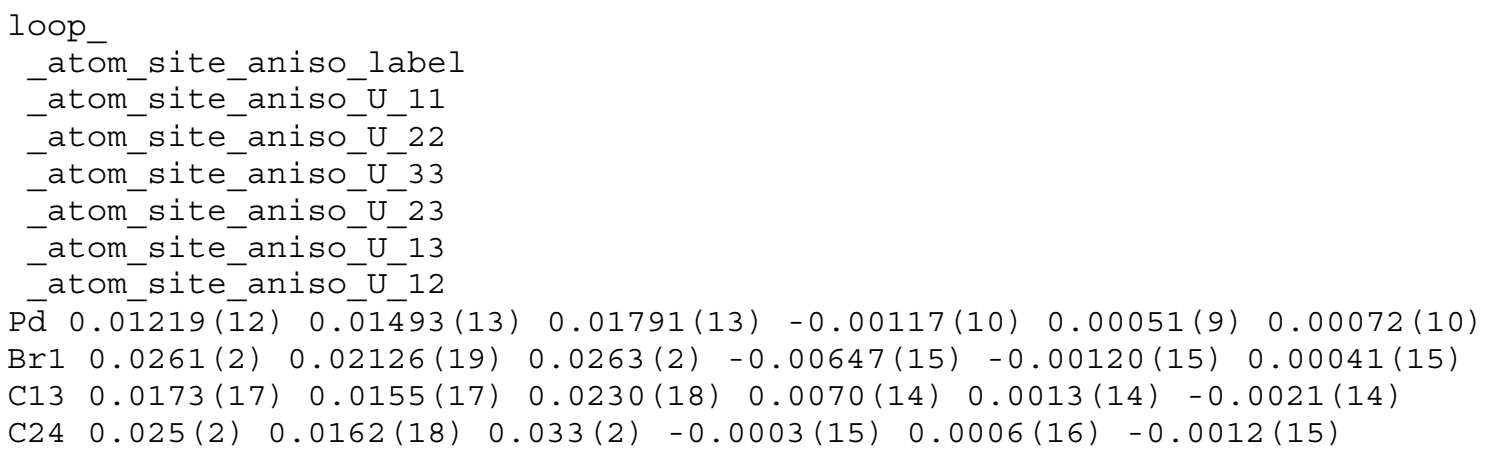




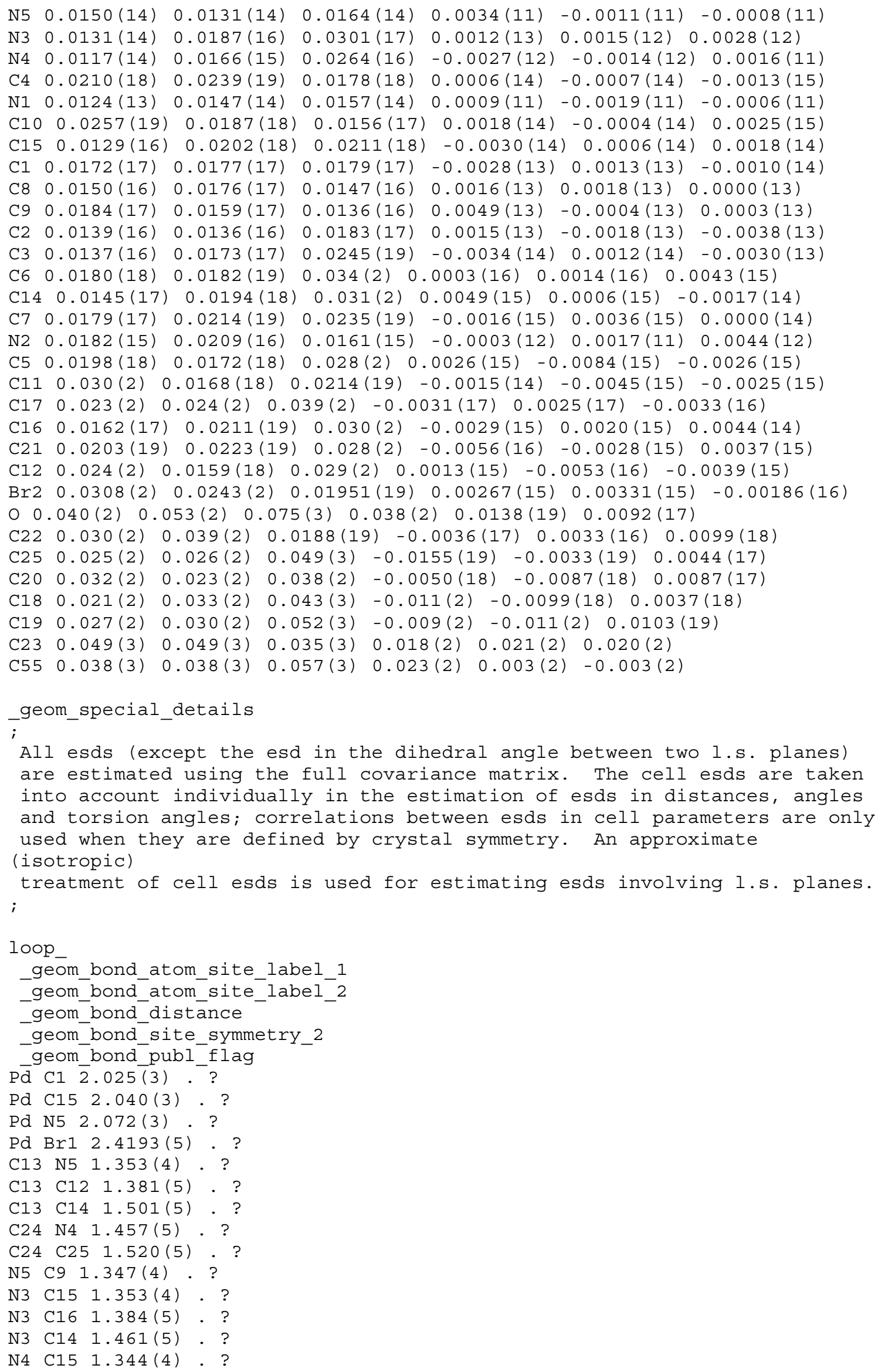




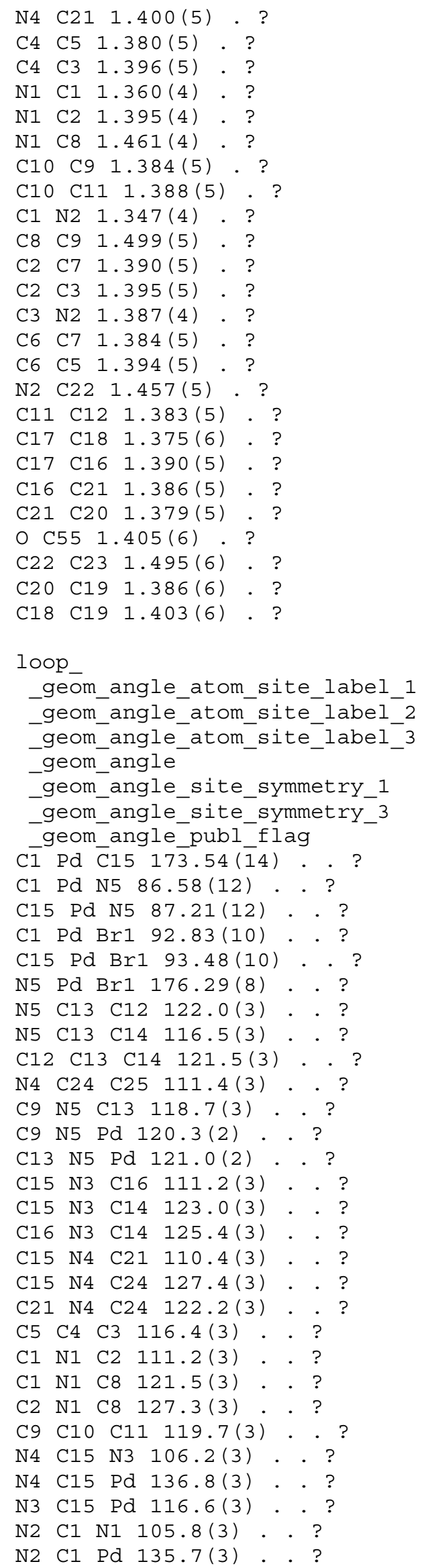




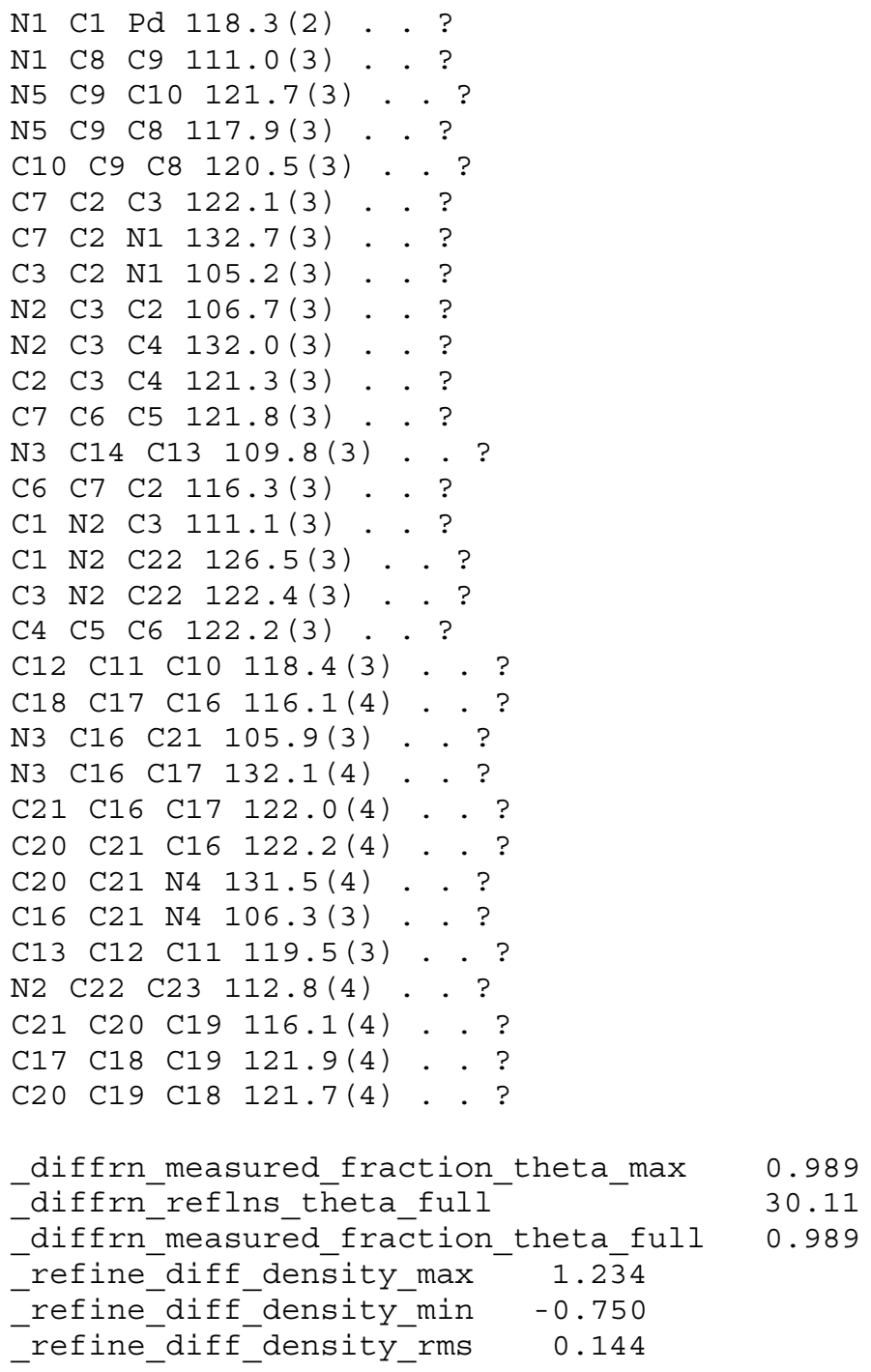

